\title{
Search for Low Mass Vector Resonances Decaying to Quark-Antiquark Pairs in Proton-Proton Collisions at $\sqrt{s}=13 \mathrm{TeV}$
}

\author{
A. M. Sirunyan et al. \\ (CMS Collaboration)
}

(Received 30 May 2017; published 15 September 2017)

\begin{abstract}
A search is reported for a narrow vector resonance decaying to quark-antiquark pairs in proton-proton collisions at $\sqrt{s}=13 \mathrm{TeV}$, collected with the CMS detector at the LHC. The data sample corresponds to an integrated luminosity of $2.7 \mathrm{fb}^{-1}$. The vector resonance is produced at large transverse momenta, with its decay products merged into a single jet. The resulting signature is a peak over background in the distribution of the invariant mass of the jet. The results are interpreted in the framework of a leptophobic vector resonance and no evidence is found for such particles in the mass range of 100-300 GeV. Upper limits at $95 \%$ confidence level on the production cross section are presented in a region of mass-coupling phase space previously unexplored at the LHC. The region below $140 \mathrm{GeV}$ has not been explored by any previous experiments.
\end{abstract}

DOI: 10.1103/PhysRevLett.119.111802

Over the past half century, searches for narrow resonances in the dijet invariant mass spectrum have been an important part of the physics program at every collider. Such searches are well motivated within the many classes of theories beyond the current standard model (SM) that predict resonances with significant couplings to quarks [1-11]. The first searches for such particles at the UA1 [12] and UA2 [13,14] experiments at the CERN $\mathrm{S} \bar{p} p \mathrm{~S}$ have been extended to larger values of resonance masses by the CDF [15-19] and D0 [20-22] experiments at the Fermilab Tevatron, and by the ATLAS [23-32] and CMS [33-45] experiments at the CERN LHC.

In this Letter, we report on a search for vector $\left(Z^{\prime}\right)$ resonances decaying to quark-antiquark pairs $(q \bar{q})$ using the data collected in $p p$ collisions at $\sqrt{s}=13 \mathrm{TeV}$ by the CMS detector, corresponding to an integrated luminosity of $2.7 \mathrm{fb}^{-1}$. This search concentrates on the mass region of $100-300 \mathrm{GeV}$, exploring for the first time masses below $140 \mathrm{GeV}[13,14]$. To access this mass regime, we present a new analysis technique exploiting novel jet substructure techniques and associated production with a jet. The search is interpreted in the framework of a leptophobic vector resonance and can also be reinterpreted in terms of searches for generic vectorlike resonances decaying to quarks [46].

With the increase in collision energy and beam intensity at hadron colliders, there has been a loss of search sensitivity for lighter resonances with couplings to

\footnotetext{
*Full author list given at the end of the article.

Published by the American Physical Society under the terms of the Creative Commons Attribution 4.0 International license. Further distribution of this work must maintain attribution to the author(s) and the published article's title, journal citation, and DOI.
}

quarks and gluons. The main experimental difficulties originate from the large increase in the cross section of multijet backgrounds at small resonance masses, and the more restrictive trigger requirements needed to reduce the data recording rate because of limited resources for event processing and storage. To overcome these difficulties, we require events to have a jet with large transverse momentum $\left(p_{T}\right)$ from initial-state radiation (ISR) produced in association with the resonance. This ISR constraint provides enough energy in the event to satisfy the trigger. Combinatorial backgrounds are reduced by requiring the resonance be reconstructed within a single jet. The jet is required to have the two-prong structure expected from signal. The dominant background is from SM events composed of jets produced through quantum chromodynamics (QCD). This multijet background is estimated by inverting the two-prong substructure requirement, which is specifically designed to be uncorrelated with the jet mass. By searching for new particles produced in association with an ISR jet, we are able to search for new resonances in a coupling and mass regime to which previous searches were insensitive.

A detailed description of the CMS detector, together with a definition of the coordinate system and the relevant kinematic variables, can be found in Ref. [47]. The central feature of the CMS apparatus is a superconducting solenoid of $6 \mathrm{~m}$ internal diameter, providing a magnetic field of $3.8 \mathrm{~T}$. A silicon pixel and strip tracker, a lead tungstate crystal electromagnetic calorimeter (ECAL), and a brass and scintillator hadron calorimeter (HCAL), each composed of a barrel and two end-cap sections, reside within the solenoid. Forward calorimeters extend the pseudorapidity $(\eta)$ [47] coverage provided by the barrel and end-cap detectors. Muons are measured in gas-ionization detectors embedded in the steel flux-return yoke outside the solenoid. 
We employ the CMS particle-flow (PF) algorithm $[48,49]$ to reconstruct and identify each individual particle through an optimized combination of information from the various elements of the CMS detector. The energy of a photon is obtained directly from the ECAL measurement, corrected for losses in detector sensitivity due to zero suppression near signal threshold. The energy of an electron is determined from a combination of its momentum at the primary interaction vertex determined using the tracker, the energy of the corresponding ECAL cluster, and the energy sum of all bremsstrahlung photons spatially compatible with originating from the electron track. The energy of a muon is obtained from the curvature of the corresponding track. The energy of a charged hadron is obtained from a combination of its momentum measured in the tracker and the matching of ECAL and HCAL energy depositions, corrected for the response function of the calorimeters to hadronic showers. The energy of a neutral hadron is obtained from the corresponding corrected ECAL and HCAL energies.

The benchmark $Z^{\prime}$ signal events are simulated at leading order (LO) using the MADGRAPH5_AMC@NLO 2.2.1 generator [50], PYTHIA 8.205 generator [51,52], and GEANT4 [53]. The dominant SM backgrounds arise from multijets, $W \rightarrow q \bar{q}$, and $Z \rightarrow q \bar{q}$ sources. These backgrounds are simulated at LO using the MADGRAPH5_AMC@NLO 2.2.1 generator and hadronized with PYTHIA using the CUETP8M1 tune [54]. To events containing $W$ and $Z$ bosons, we apply higherorder QCD and electroweak (EWK) corrections to improve modeling of the high- $p_{T} W$ and $Z$ events, following [5559]. Owing to the similarity between the signal process and the SM $W$ and $Z$ topologies, the next-to-leading-order (NLO) QCD corrections and associated uncertainties applied to the $W$ and $Z$ simulations are also applied to the signal simulation. The NLO EWK corrections are not applied since such corrections are different for leptophobic $Z^{\prime}$ signal production.

To isolate the $Z^{\prime}$ signal and overcome trigger restrictions, we select a high- $p_{T}$ signal jet, which typically recoils against another high- $p_{T}$ ISR jet. We use a logical "or" of trigger requirements that selects on the total hadronic transverse energy in the event and, in some cases, additionally selects on the mass of the jet after removing remnants of soft radiation, with the jet-trimming technique [60]. Jets at the trigger level are reconstructed using the anti- $k_{T}$ algorithm $[61,62]$ with a distance parameter of 0.8 , and are referred to as AK8 jets.

After the trigger selection, the AK8 jets are reconstructed by clustering particle candidates in the event. To mitigate the effect of additional interactions in the same or adjacent bunch crossings (pileup), the pileup per particle identification algorithm [63] is used, prior to jet clustering, to weight the PF candidates on the likelihood of coming from the primary interaction vertex. Other corrections are applied to jet energies as a function of jet $\eta$ and $p_{T}$ to match the detector response and to bring data and simulation into agreement. We require at least one AK8 jet to have $p_{T}>$ $500 \mathrm{GeV}$ and to satisfy $|\eta|<2.5$ to be fully efficient with respect to the trigger requirements. We veto events containing identified and isolated electrons or muons with $p_{T}>10 \mathrm{GeV}$, and $|\eta|<2.5$ or 2.4 , respectively, to reduce backgrounds from SM EWK processes.

The Lorentz-boosted $Z^{\prime} \rightarrow q \bar{q}$ system is reconstructed as a single high- $p_{T}$ jet, where the decay products have merged into one object, assumed to be the jet of highest $p_{T}$ in the event. From simulation studies, approximately $70 \%$ of $Z^{\prime}$ signal events satisfy this assumption. The most important variable is the soft-drop jet mass $\left(m_{\mathrm{SD}}\right)$, which employs soft-drop grooming [64,65], a technique used to remove soft and wide-angle radiation in jets. The variable $m_{\mathrm{SD}}$ enhances the peak at the $Z^{\prime}$ mass for signal events and the same act of soft-drop grooming reduces the masses from background quark- and gluon-initiated events. For computing $m_{\mathrm{SD}}$, we use a soft radiation fraction selection greater than $z=0.1$ and an angular exponent parameter of $\beta=0$.

Since the $Z^{\prime} \rightarrow q \bar{q}$ jet has a two-prong structure, we use substructure tools developed to identify jets from hadronically decaying $W$ bosons. We use a variable based on the $N$-subjettiness ratio $\tau_{2} / \tau_{1}$ [66], also referred to as $\tau_{21}$, which is used in other searches to determine a jet's consistency with having a two-prong structure [67-69]. However, any chosen cutoff $\tau_{21}$ value sculpts the jet mass distributions in a way that depends on the $p_{T}$ of the jet, and causes a complex mass distribution that peaks at high jet mass. This limits its usefulness when searching for resonant peaks over a large range in $p_{T}$. We therefore employ a transformation of $\tau_{21}$ to $\tau_{21}^{\mathrm{DDT}}$, where DDT stands for designed decorrelated tagger [70]. It is a generic technique to reduce mass correlations of the observable in multijet events. We specifically deploy it here through a linear transformation between $\tau_{21}$ and a dimensionless jet mass variable [71], $\rho^{\mathrm{DDT}}=\ln \left[m_{\mathrm{SD}}^{2} /\left(\mu_{0} p_{T}\right)\right]$, where the scale $\mu_{0}=1 \mathrm{GeV}$. We determine the optimal transformation from simulation to be $\tau_{21}^{\mathrm{DDT}}=\tau_{21}+0.063 \rho^{\mathrm{DDT}}$. Events are selected by requiring $\tau_{21}^{\mathrm{DDT}}<0.38$.

The dominant background comes from multijet events. For each $Z^{\prime}$ mass point, we estimate this background using sidebands of kinematic distributions in the data, in order to minimize reliance on the simulation. We use both $\rho^{\mathrm{DDT}}$ and $\tau_{21}^{\mathrm{DDT}}$ background-dominated sideband regions and take advantage of the lack of correlation between $\tau_{21}^{\text {DDT }}$ and $\rho^{\mathrm{DDT}}$. The method is illustrated in Fig. 1 (left), where the mean in the distribution of $\tau_{21}^{\mathrm{DDT}}$ is plotted as a function of $\rho^{\mathrm{DDT}}$ for different bins in jet $p_{T}$. Both $\tau_{21}^{\mathrm{DDT}}$ and $p_{T}$ have little dependence on $\rho^{\mathrm{DDT}}$ in range from 0 to 4 corresponding to $30 \mathrm{GeV}<m_{\mathrm{SD}}<300 \mathrm{GeV}$. Figure 1 (right) illustrates the strategy for estimating the multijet background. We define the pass-to-fail ratio, $R_{p / f}$, as the ratio of yields in the pass region $\left(\tau_{21}^{\mathrm{DDT}}<0.38\right)$ to the yields in the fail 

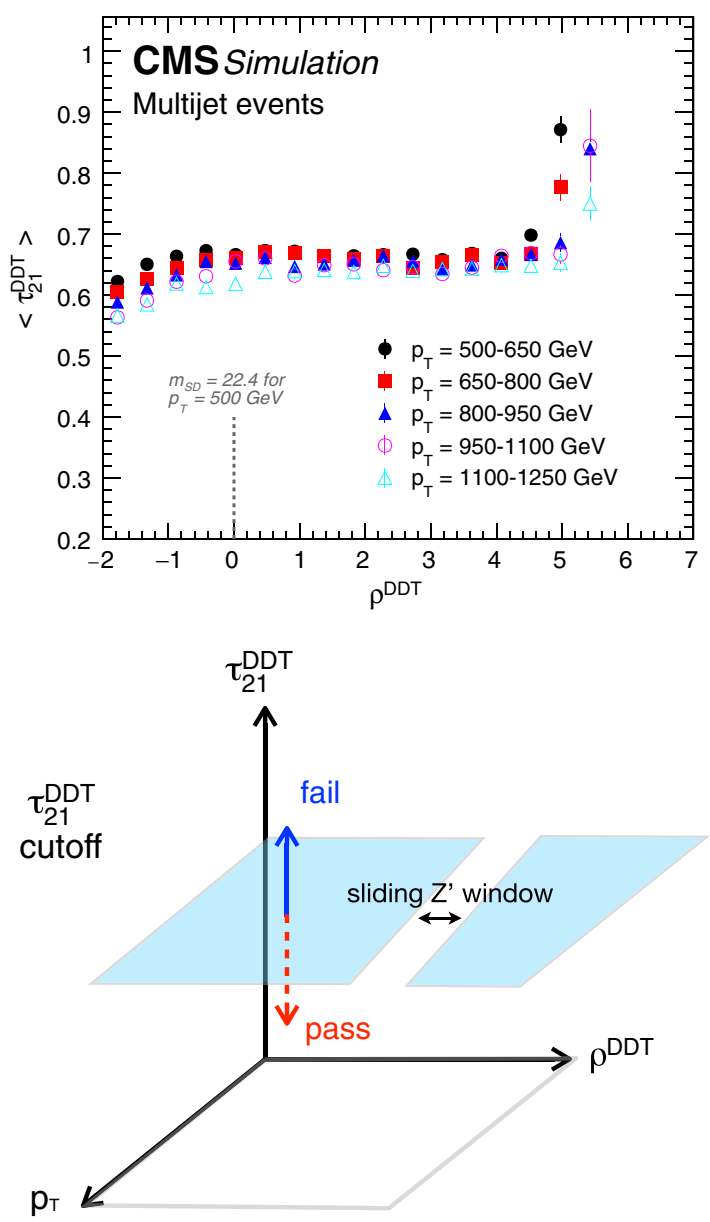

FIG. 1. The average of $\tau_{21}^{\text {DDT }}$ versus $\rho^{\text {DDT }}$ for different bins in jet $p_{T}$ (upper). A schematic of the method used to estimate the background (lower). After a $\tau_{21}^{\mathrm{DDT}}$ selection is applied, the multijet event distributions in the passing region are predicted from their distributions in the failing region with the use of a pass-to-fail ratio extracted through a two-dimensional fit in $\left(\rho^{\mathrm{DDT}}, p_{T}\right)$ space. Events in a sliding window around the desired $Z^{\prime}$ mass point are vetoed when determining the pass-to-fail ratio.

region $\left(\tau_{21}^{\mathrm{DDT}}>0.38\right)$. We rescale multijet events from the fail region using $R_{p / f}$ parameterized in $p_{T}$ and $\rho^{\mathrm{DDT}}$ to predict the mulijet signal region background,

$P_{\text {pass }}^{\text {multijet }}\left(p_{T}, \rho^{\mathrm{DDT}}\right)=R_{p / f}\left(p_{T}, \rho^{\mathrm{DDT}}\right) P_{\text {fail }}^{\text {multijet }}\left(p_{T}, \rho^{\mathrm{DDT}}\right)$,

where $P_{\text {pass }}^{\text {multijet }}$ and $P_{\text {fail }}^{\text {multijet }}$ are the passing and failing multijet distributions. We determine $R_{p / f}$ by fitting the ratio of data events in the pass to fail regions over the $\left(\rho^{\mathrm{DDT}}, p_{T}\right)$ space. With this method, we fit for any residual correlations between $\tau_{21}^{\mathrm{DDT}}$ and $\rho^{\mathrm{DDT}}$, modeling them as polynomial functions. Fitting $R_{p / f}$, in place of the normalization, allows the region where the trigger selection is not fully efficient to be exploited; therefore, we begin the $R_{p / f}$ fit in this region at a jet $p_{T}>350 \mathrm{GeV}$. Prior to performing the fit, we subtract the $W$ and $Z$ boson contributions expected from simulation, at NLO, to estimate only nonresonant contributions such as the multijet background. To avoid a bias induced by a given $Z^{\prime}$ signal, we exclude a window from the $R_{p / f}$ fit centered about the given $Z^{\prime} m_{\mathrm{SD}}$ of width $\pm 10 \% m_{Z^{\prime}}$, which corresponds approximately to the jet mass resolution. The missing strip in the $R_{p / f}$ shape in Fig. 1 (right) reflects this window. Fits are therefore performed as a function of $Z^{\prime}$ mass to estimate the background. The two-dimensional $R_{p / f}$ depends slightly on the chosen mass hypothesis, as different windows in jet mass are excluded for each $Z^{\prime}$ mass. Finally, the mass distribution in data is tested for the presence of signal using the multijet prediction along with the simulated $W$ and $Z$ predictions.

The uncertainties in the method described above arise from three sources. The first uncertainty arises from residual systematic effects in the method, as determined from a self-consistency check in simulated events. This systematic effect is correlated across all jet mass bins. The second is due to the uncertainty in the $R_{p / f}$ shape from the fit parameter uncertainties, also correlated across all jet mass bins. The third source originates from uncorrelated statistical uncertainties in the size of the fail sample and the statistical uncertainties in the self-consistency test.

Smaller backgrounds from resonant SM processes $(W / Z+$ jets $)$ are estimated from simulation, but with corrections for the mass shapes and efficiencies evaluated from data as discussed below. The expectations for all SM background processes (multijets, $W / Z+$ jets) are determined for jet masses from 30 to $330 \mathrm{GeV}$. Including the region near the $W$ and $Z$ boson masses provides a constraint on the mass distribution of the $Z^{\prime}$ and its normalization by determining the $W / Z+$ jets contribution in situ.

The systematic effects on shapes and normalizations of the $W, Z$, and signal distributions are correlated. We constrain the jet mass scale, jet mass resolution, and $\tau_{21}^{\mathrm{DDT}}$ selection efficiency using a sample of merged $W$ boson jets in semileptonic $t \bar{t}$ events in data. Using the same $\tau_{21}^{\mathrm{DDT}}$ selection as in the analysis provides passing and failing regions for merged $W$ boson jets in data and in simulation. A simultaneous fit to the two samples in $m_{\mathrm{SD}}$ is performed to extract the tagging efficiency of a merged $W$ boson jet, its jet mass scale, and the resolution in simulation and in data. Scale factors relating data and simulation are then computed and applied to the simulation. These scale factors and their uncertainties determine the initial distributions for the $W, Z$, and signal that are further constrained in the final fit in situ using the presence of the $W$ and $Z$ peaks in the jet mass distribution. Finally, additional systematic uncertainties are applied to the $W, Z$, and $Z^{\prime}$ signal yields that are associated with higher-order corrections to the boson $p_{T}$ distributions, jet energy scale [72], the modeling of pileup, and the integrated beam luminosity [73]. A quantitative summary of the systematic effects considered is shown in Table I. 
TABLE I. Summary of the systematic uncertainties for signal and background. Uncertainties due to the finite size of the simulated samples are applied only to the $Z^{\prime}$ signal. Electroweak uncertainties are applied only to the $W / Z$ boson processes. Dashes (-) indicate the uncertainty does not apply.

\begin{tabular}{lcc}
\hline \hline Systematic effect & Multijet & $\mathrm{SM} W / Z$ and $Z^{\prime}$ \\
\hline Multijet fit parameters & $1 \%-4 \%$ & $\ldots$ \\
Multijet bin-by-bin statistics & $1 \%-20 \%$ & $\ldots$ \\
Multijet fit closure & $1 \%-12 \%$ & $\ldots$ \\
$W$ boson tagging scale factor & $\ldots$ & $20 \%$ \\
NLO QCD $p_{\mathrm{T}}$ corrections & $\ldots$ & $8 \%-12 \%$ \\
Jet energy and mass resolution & $\ldots$ & $10 \%$ \\
Simulation sample size & $\ldots$ & $1 \%-30 \%$ \\
Integated luminosity & $\ldots$ & $2.7 \%$ \\
Pileup & $\ldots$ & $<1 \%$ \\
Jet energy and mass scale & $\ldots$ & $1 \%$ \\
\hline \hline
\end{tabular}

To validate the background estimation method and its associated systematic uncertainties, studies are performed on simulated events by injecting a signal and testing for a bias in the extracted signal cross section. No significant bias is observed. We combine the estimates of various $\mathrm{SM}$ background processes, and search for a $Z^{\prime}$ resonance in the $100-300 \mathrm{GeV}$ mass range, while the SM backgrounds are estimated in the $30-330 \mathrm{GeV}$ mass range. Figure 2 shows the soft-drop jet mass distribution with a background calculated for the $Z^{\prime}$ search at a mass of $135 \mathrm{GeV}$. Expected background contributions from the $W$ and $Z$ bosons are clearly visible in the data.

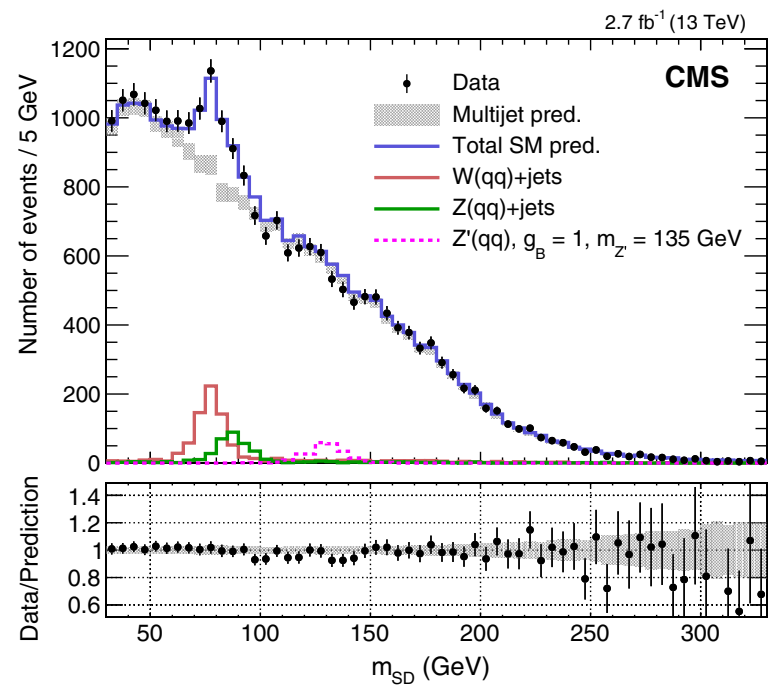

FIG. 2. Soft-drop jet mass distribution after final selection. Data are shown as black points. The multijet background, including uncertainties, is shown as gray boxes, while the sum of the SM processes is given by the blue line. Contributions from the SM $W / Z$ boson and a hypothetical $Z^{\prime}$ signal at a mass of $135 \mathrm{GeV}$ are also indicated. In the bottom panel, we show the ratio of the data to the background prediction, including uncertainties.
The number of events in data is consistent with the predicted backgrounds from SM processes. Results are obtained from combined signal and background binned likelihood fits to the jet mass distribution, such as the one shown in Fig. 2. The results are interpreted as upper limits on the production cross section for signal in terms of $95 \%$ confidence level (C.L.) upper limits computed using the modified frequentist approach $\left(\mathrm{CL}_{\mathrm{s}}\right)$, taking the likelihood as the test statistic $[74,75]$ in the asymptotic approximation [76]. Systematic uncertainties are treated as nuisance parameters that are modeled through log-normal priors. The probability of the downward fluctuation of the data at a $Z^{\prime}$ signal mass of $100 \mathrm{GeV}$ is less than $0.15 \%$ and corresponds to a local $2.8 \sigma$ effect. We attribute this to a statistical fluctuation in data. Studies of the background model are performed by injecting simulated signal events and we find negligible biases in the fitted signal strength. An alternative background estimate is also studied by fitting directly the jet mass distribution; results are consistent within $1 \sigma$.

Upper limits on signal cross sections are translated into the coupling of the $Z^{\prime}$ boson to quarks $\left(g_{q}\right)$ as a function of $m_{Z^{\prime}}$. In Fig. 3, we compare our results with previous results from the UA2, CDF, ATLAS, and CMS experiments. The limits from the UA2 and CDF experiments, and the indirect constraints from the $Z \rightarrow q \bar{q}$ width, are obtained following the approach described in Ref. [77]. We exclude coupling values of $g_{q}$ from 0.071 to 0.260 over the selected $Z^{\prime}$ mass range.

In summary, a search by CMS for a vector $\left(Z^{\prime}\right)$ resonance decaying to a $q \bar{q}$ pair in the mass range from $100-300 \mathrm{GeV}$ has been presented. The results are interpreted in the framework of a leptophobic vector resonance model. No excess above the SM prediction is observed, and 95\%

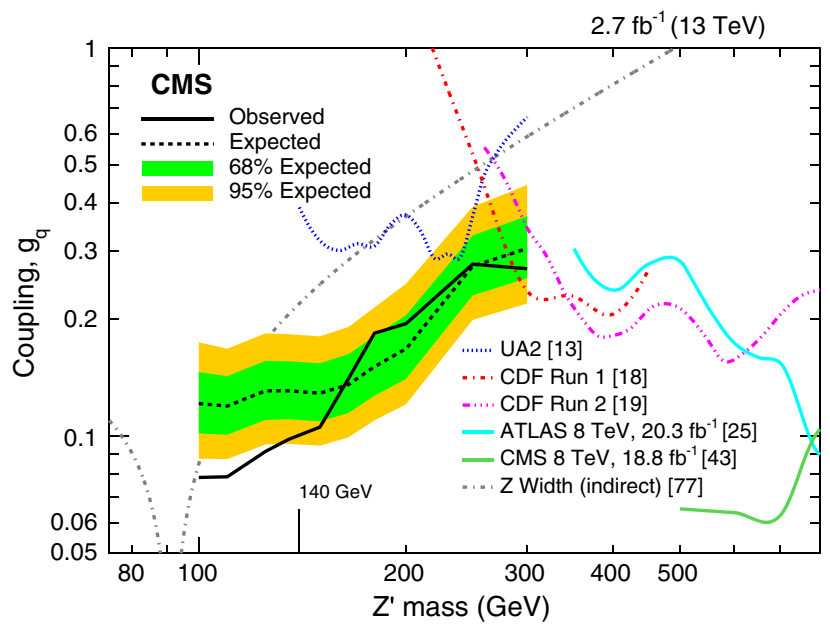

FIG. 3. Upper limits on $g_{q}$ as a function of the $Z^{\prime}$ mass at $95 \%$ C.L. [78]. Limits from other relevant searches are shown. An indirect constraint from the width of the SM $Z$ boson is also given. 
confidence level upper limits are set on the coupling of the $Z^{\prime}$ to quarks as a function of the $Z^{\prime}$ mass. These limits are the most stringent to date for masses less than $300 \mathrm{GeV}$. For masses below $140 \mathrm{GeV}$, these are the only available direct limits.

We congratulate our colleagues in the CERN accelerator departments for the excellent performance of the LHC and thank the technical and administrative staffs at CERN and at other CMS institutes for their contributions to the success of the CMS effort. In addition, we gratefully acknowledge the computing centers and personnel of the Worldwide LHC Computing Grid for delivering so effectively the computing infrastructure essential to our analyses. Finally, we acknowledge the enduring support for the construction and operation of the LHC and the CMS detector provided by the following funding agencies: BMWFW and FWF (Austria); FNRS and FWO (Belgium); CNPq, CAPES, FAPERJ, and FAPESP (Brazil); MES (Bulgaria); CERN; CAS, MoST, and NSFC (China); COLCIENCIAS (Colombia); MSES and CSF (Croatia); RPF (Cyprus); SENESCYT (Ecuador); MoER, ERC IUT, and ERDF (Estonia); Academy of Finland, MEC, and HIP (Finland); CEA and CNRS/IN2P3 (France); BMBF, DFG, and HGF (Germany); GSRT (Greece); OTKA and NIH (Hungary); DAE and DST (India); IPM (Iran); SFI (Ireland); INFN (Italy); MSIP and NRF (Republic of Korea); LAS (Lithuania); MOE and UM (Malaysia); BUAP, CINVESTAV, CONACYT, LNS, SEP, and UASLP-FAI (Mexico); MBIE (New Zealand); PAEC (Pakistan); MSHE and NSC (Poland); FCT (Portugal); JINR (Dubna); MON, RosAtom, RAS, RFBR, and RAEP (Russia); MESTD (Serbia); SEIDI, CPAN, PCTI, and FEDER (Spain); Swiss Funding Agencies (Switzerland); MST (Taipei); ThEPCenter, IPST, STAR, and NSTDA (Thailand); TUBITAK and TAEK (Turkey); NASU and SFFR (Ukraine); STFC (United Kingdom); DOE and NSF (USA).

[1] E. Eichten, I. Hinchliffe, K. D. Lane, and C. Quigg, Supercollider physics, Rev. Mod. Phys. 56, 579 (1984).

[2] P. H. Frampton and S. L. Glashow, Chiral color: An alternative to the standard model, Phys. Lett. B 190, 157 (1987).

[3] U. Baur, I. Hinchliffe, and D. Zeppenfeld, Excited quark production at hadron colliders, Int. J. Mod. Phys. A 02, 1285 (1987).

[4] J. L. Hewett and T. G. Rizzo, Low-energy phenomenology of superstring inspired E(6) models, Phys. Rep. 183, 193 (1989).

[5] U. Baur, M. Spira, and P. M. Zerwas, Excited quark and lepton production at hadron colliders, Phys. Rev. D 42, 815 (1990).

[6] E. H. Simmons, Coloron phenomenology, Phys. Rev. D 55, 1678 (1997).

[7] L. Randall and R. Sundrum, An Alternative to Compactification, Phys. Rev. Lett. 83, 4690 (1999).
[8] S. Cullen, M. Perelstein, and M. E. Peskin, TeV strings and collider probes of large extra dimensions, Phys. Rev. D 62, 055012 (2000).

[9] L. A. Anchordoqui, H. Goldberg, D. Lust, S. Nawata, S. Stieberger, and T. R. Taylor, Dijet Signals for Low Mass Strings at the Large Hadron Collider, Phys. Rev. Lett. 101, 241803 (2008).

[10] T. Han, I. Lewis, and Z. Liu, Colored resonant signals at the LHC: Largest rate and simplest topology, J. High Energy Phys. 12 (2010) 085.

[11] R. S. Chivukula, A. Farzinnia, E. H. Simmons, and R. Foadi, Production of massive color-octet vector bosons at next-to-leading order, Phys. Rev. D 85, 054005 (2012).

[12] C. Albajar et al. (UA1 Collaboration), Two-jet mass distributions at the CERN proton-antiproton collider, Phys. Lett. B 209, 127 (1988).

[13] J. Alitti et al. (UA2 Collaboration), A measurement of two jet decays of the $W$ and $Z$ bosons at the CERN $\bar{p} p$ collider, Z. Phys. C 49, 17 (1991).

[14] J. Alitti et al. (UA2 Collaboration), A search for new intermediate vector mesons and excited quarks decaying to two jets at the CERN $\bar{p} p$ collider, Nucl. Phys. B400, 3 (1993).

[15] F. Abe et al. (CDF Collaboration), The two jet invariant mass distribution at $\sqrt{s}=1.8 \mathrm{TeV}$, Phys. Rev. D 41, 1722 (1990).

[16] F. Abe et al. (CDF Collaboration), Search for Quark Compositeness, Axigluons and Heavy Particles Using the Dijet Invariant Mass Spectrum Observed in $p \bar{p}$ Collisions, Phys. Rev. Lett. 71, 2542 (1993).

[17] F. Abe et al. (CDF Collaboration), Search for New Particles Decaying to Dijets in $p \bar{p}$ Collisions at $\sqrt{s}=1.8 \mathrm{TeV}$, Phys. Rev. Lett. 74, 3538 (1995).

[18] F. Abe et al. (CDF Collaboration), Search for new particles decaying to dijets at CDF, Phys. Rev. D 55, R5263 (1997).

[19] T. Aaltonen et al. (CDF Collaboration), Search for new particles decaying into dijets in proton-antiproton collisions at $\sqrt{s}=1.96 \mathrm{TeV}$, Phys. Rev. D 79, 112002 (2009).

[20] B. Abbott et al. (D0 Collaboration), Measurement of Dijet Angular Distributions and Search for Quark Compositeness, Phys. Rev. Lett. 80, 666 (1998).

[21] B. Abbott et al. (D0 Collaboration), The Dijet Mass Spectrum and a Search for Quark Compositeness in $\bar{p} p$ Collisions at $\sqrt{s}=1.8 \mathrm{TeV}$, Phys. Rev. Lett. 82, 2457 (1999).

[22] V. M. Abazov et al. (D0 Collaboration), Search for new particles in the two jet decay channel with the D0 detector, Phys. Rev. D 69, 111101 (2004).

[23] ATLAS Collaboration, Search for New Particles in Two-Jet Final States in $7 \mathrm{TeV}$ Proton-Proton Collisions with the ATLAS Detector at the LHC, Phys. Rev. Lett. 105, 161801 (2010).

[24] ATLAS Collaboration, Search for quark contact interactions in dijet angular distributions in pp collisions at $\sqrt{s}=7 \mathrm{TeV}$ measured with the ATLAS detector, Phys. Lett. B 694, 327 (2011).

[25] ATLAS Collaboration, A search for new physics in dijet mass and angular distributions in pp collisions at $\sqrt{s}=$ $7 \mathrm{TeV}$ measured with the ATLAS detector, New J. Phys. 13, 053044 (2011). 
[26] ATLAS Collaboration, Search for new physics in the dijet mass distribution using $1 \mathrm{fb}^{-1}$ of pp collision data at $\sqrt{\mathrm{s}}=$ $7 \mathrm{TeV}$ collected by the ATLAS detector, Phys. Lett. B 708, 37 (2012).

[27] ATLAS Collaboration, ATLAS search for new phenomena in dijet mass and angular distributions using pp collisions at $\sqrt{s}=7 \mathrm{TeV}$, J. High Energy Phys. 01 (2013) 029.

[28] ATLAS Collaboration, Search for new phenomena in the dijet mass distribution using pp collision data at $\sqrt{s}=$ $8 \mathrm{TeV}$ with the ATLAS detector, Phys. Rev. D 91, 052007 (2015).

[29] ATLAS Collaboration, Search for New Phenomena in Dijet Angular Distributions in Proton-Proton Collisions at $\sqrt{s}=8 \mathrm{TeV}$ Measured with the ATLAS Detector, Phys. Rev. Lett. 114, 221802 (2015).

[30] ATLAS Collaboration, Search for new phenomena in dijet mass and angular distributions from pp collisions at $\sqrt{\mathrm{s}}=$ $13 \mathrm{TeV}$ with the ATLAS detector, Phys. Lett. B 754, 302 (2016).

[31] ATLAS Collaboration, Search for resonances in the mass distribution of jet pairs with one or two jets identified as b-jets in proton-proton collisions at $\sqrt{s}=13 \mathrm{TeV}$ with the ATLAS detector, Phys. Lett. B 759, 229 (2016).

[32] ATLAS Collaboration, Search for new phenomena in dijet events using $37 \mathrm{fb}^{-1}$ of pp collision data collected at $\sqrt{s}=$ $13 \mathrm{TeV}$ with the ATLAS detector, arXiv:1703.09127.

[33] CMS Collaboration, Search for Dijet Resonances in $7 \mathrm{TeV}$ pp Collisions at CMS, Phys. Rev. Lett. 105, 211801 (2010); Errattum, Phys. Rev. Lett.106, 029902(E) (2011).

[34] CMS Collaboration, Search for Quark Compositeness with the Dijet Centrality Ratio in pp Collisions at $\sqrt{s}=7 \mathrm{TeV}$, Phys. Rev. Lett. 105, 262001 (2010).

[35] CMS Collaboration, Measurement of Dijet Angular Distributions and Search for Quark Compositeness in pp Collisions at $\sqrt{s}=7 \mathrm{TeV}$, Phys. Rev. Lett. 106, 201804 (2011).

[36] CMS Collaboration, Search for resonances in the dijet mass spectrum from $7 \mathrm{TeV}$ pp collisions at CMS, Phys. Lett. B 704, 123 (2011).

[37] CMS Collaboration, Search for quark compositeness in dijet angular distributions from pp collisions at $\sqrt{s}=7 \mathrm{TeV}$, J. High Energy Phys. 05 (2012) 055.

[38] CMS Collaboration, Search for narrow resonances and quantum black holes in inclusive and b-tagged dijet mass spectra from pp collisions at $\sqrt{s}=7 \mathrm{TeV}$, J. High Energy Phys. 01 (2013) 013.

[39] CMS Collaboration, Search for narrow resonances using the dijet mass spectrum in pp collisions at $\sqrt{s}=8 \mathrm{TeV}$, Phys. Rev. D 87, 114015 (2013).

[40] CMS Collaboration, Search for quark contact interactions and extra spatial dimensions using dijet angular distributions in proton-proton collisions at $\sqrt{s}=8 \mathrm{TeV}$, Phys. Lett. B 746, 79 (2015).

[41] CMS Collaboration, Search for resonances and quantum black holes using dijet mass spectra in proton-proton collisions at $\sqrt{s}=8 \mathrm{TeV}$, Phys. Rev. D 91, 052009 (2015).

[42] CMS Collaboration, Search for Narrow Resonances Decaying to Dijets in Proton-Proton Collisions at $\sqrt{s}=13 \mathrm{TeV}$, Phys. Rev. Lett. 116, 071801 (2016).
[43] CMS Collaboration, Search for Narrow Resonances in Dijet Final States at $\sqrt{s}=8 \mathrm{TeV}$ with the Novel CMS Technique of Data Scouting, Phys. Rev. Lett. 117, 031802 (2016).

[44] CMS Collaboration, Search for new physics with dijet angular distributions in proton-proton collisions at $\sqrt{s}=13 \mathrm{TeV}$, J. High Energy Phys. 07 (2017) 013.

[45] CMS Collaboration, Search for dijet resonances in protonproton collisions at $\sqrt{s}=13 \mathrm{TeV}$ and constraints on dark matter and other models, Phys. Lett. B 769, 520 (2016).

[46] B. A. Dobrescu and F. Yu, Coupling-mass mapping of dijet peak searches, Phys. Rev. D 88, 035021 (2013); Errattum, Phys. Rev. D90, 079901(E) (2014).

[47] CMS Collaboration, The CMS experiment at the CERN LHC, J. Instrum. 3, S08004 (2008).

[48] CMS Collaboration, CMS Report No. CMS-PAS-PFT-09001, 2009, https://cds.cern.ch/record/1194487.

[49] CMS Collaboration, CMS Report No. CMS-PAS-PFT-10001, 2010, http://cds.cern.ch/record/1247373.

[50] J. Alwall, R. Frederix, S. Frixione, V. Hirschi, F. Maltoni, O. Mattelaer, H. S. Shao, T. Stelzer, P. Torrielli, and M. Zaro, The automated computation of tree-level and next-toleading order differential cross sections, and their matching to parton shower simulations, J. High Energy Phys. 07 (2014) 079.

[51] T. Sjöstrand, S. Mrenna, and P. Skands, PYTHIA 6.4 physics and manual, J. High Energy Phys. 05 (2006) 026.

[52] T. Sjöstrand, S. Mrenna, and P. Skands, A brief introduction to PYTHIA 8.1, Comput. Phys. Commun. 178, 852 (2008).

[53] S. Agostinelli et al. (GEANT4 Collaboration), GEANT4-a simulation toolkit, Nucl. Instrum. Methods Phys. Res., Sect. A 506, 250 (2003).

[54] CMS Collaboration, Event generator tunes obtained from underlying event and multiparton scattering measurements, Eur. Phys. J. Spec. Top. 76, 155 (2016).

[55] CMS Collaboration, Search for dark matter produced with an energetic jet or a hadronically decaying $\mathrm{W}$ or $\mathrm{Z}$ boson at $\sqrt{s}=13 \mathrm{TeV}$, J. High Energy Phys. 07 (2017) 014.

[56] S. Kallweit, J. M. Lindert, P. Maierhöfer, S. Pozzorini, and M. Schönherr, NLO electroweak automation and precise predictions for $\mathrm{W}+$ multijet production at the LHC, J. High Energy Phys. 04 (2015) 012.

[57] S. Kallweit, J. M. Lindert, P. Maierhofer, S. Pozzorini, and M. Schönherr, NLO QCD+EW predictions for V+jets including off-shell vector-boson decays and multijet merging, J. High Energy Phys. 04 (2016) 021.

[58] S. Kallweit, J. M. Lindert, S. Pozzorini, M. Schönherr, and P. Maierhöfer, in Proceedings, 50th Rencontres de Moriond, QCD and High Energy Interactions (2015), p. 121, https:// arxiv.org/abs/1505.05704.

[59] M. Schönherr, S. Kallweit, J. M. Lindert, S. Pozzorini, and P. Maierhöfer, Proc. Sci., LHCP2016 (2016) 058.

[60] D. Krohn, J. Thaler, and L.-T. Wang, Jet trimming, J. High Energy Phys. 02 (2010) 084.

[61] M. Cacciari, G. P. Salam, and G. Soyez, The anti- $k_{t}$ jet clustering algorithm, J. High Energy Phys. 04 (2008) 063.

[62] M. Cacciari, G. P. Salam, and G. Soyez, FastJet user manual, Eur. Phys. J. C 72, 1896 (2012).

[63] D. Bertolini, P. Harris, M. Low, and N. Tran, Pileup per particle identification, J. High Energy Phys. 10 (2014) 059. 
[64] M. Dasgupta, A. Fregoso, S. Marzani, and G. P. Salam, Towards an understanding of jet substructure, J. High Energy Phys. 09 (2013) 029.

[65] A. J. Larkoski, S. Marzani, G. Soyez, and J. Thaler, Soft drop, J. High Energy Phys. 05 (2014) 146.

[66] J. Thaler and K. Van Tilburg, Identifying boosted objects with N-subjettiness, J. High Energy Phys. 03 (2011) 015.

[67] CMS Collaboration, Identification techniques for highly boosted W bosons that decay into hadrons, J. High Energy Phys. 12 (2014) 017.

[68] CMS Collaboration, Search for massive resonances decaying into pairs of boosted bosons in semi-leptonic final states at $\sqrt{s}=8 \mathrm{TeV}$, J. High Energy Phys. 08 (2014) 174.

[69] CMS Collaboration, Search for massive resonances in dijet systems containing jets tagged as $\mathrm{W}$ or $\mathrm{Z}$ boson decays in $\mathrm{pp}$ collisions at $\sqrt{s}=8 \mathrm{TeV}$, J. High Energy Phys. 08 (2014) 173.

[70] J. Dolen, P. Harris, S. Marzani, S. Rappoccio, and N. Tran, Thinking outside the ROCs: Designing decorrelated taggers (DDT) for jet substructure, J. High Energy Phys. 05 (2016) 156.
[71] G. P. Salam, Towards jetography, Eur. Phys. J. C 67, 637 (2010).

[72] CMS Collaboration, Determination of jet energy calibration and transverse momentum resolution in CMS, J. Instrum. 6, P11002 (2011).

[73] CMS Collaboration, CMS Report No. CMS-PAS-LUM-15001, 2016, https://cds.cern.ch/record/2138682.

[74] T. Junk, Confidence level computation for combining searches with small statistics, Nucl. Instrum. Methods Phys. Res., Sect. A 434, 435 (1999).

[75] A. L. Read, Presentation of search results: The $C L_{s}$ technique, J. Phys. G 28, 2693 (2002).

[76] G. Cowan, K. Cranmer, E. Gross, and O. Vitells, Asymptotic Formulae for Likelihood-Based Tests of New Physics, Eur. Phys. J. C 71, 1554 (2011); Erratum, Eur. Phys. J. C 73, 2501 (2013).

[77] B. A. Dobrescu and C. Frugiuele, Hidden GeV-Scale Interactions of Quarks, Phys. Rev. Lett. 113, 061801 (2014).

[78] G. Busoni et al., Recommendations on presenting LHC searches for missing transverse energy signals using simplified $s$-channel models of dark matter, arXiv:1603.04156.

A. M. Sirunyan, ${ }^{1}$ A. Tumasyan, ${ }^{1}$ W. Adam, ${ }^{2}$ F. Ambrogi, ${ }^{2}$ E. Asilar, ${ }^{2}$ T. Bergauer, ${ }^{2}$ J. Brandstetter, ${ }^{2}$ E. Brondolin, ${ }^{2}$ M. Dragicevic, ${ }^{2}$ J. Erö, ${ }^{2}$ M. Flechl, ${ }^{2}$ M. Friedl, ${ }^{2}$ R. Frühwirth, ${ }^{2, b}$ V. M. Ghete, ${ }^{2}$ J. Grossmann, ${ }^{2}$ J. Hrubec, ${ }^{2}$ M. Jeitler, ${ }^{2, b}$ A. König, ${ }^{2}$ N. Krammer, ${ }^{2}$ I. Krätschmer, ${ }^{2}$ D. Liko, ${ }^{2}$ T. Madlener, ${ }^{2}$ I. Mikulec, ${ }^{2}$ E. Pree, ${ }^{2}$ D. Rabady, ${ }^{2}$ N. Rad, ${ }^{2}$ H. Rohringer, ${ }^{2}$ J. Schieck, ${ }^{2, b}$ R. Schöfbeck, ${ }^{2}$ M. Spanring, ${ }^{2}$ D. Spitzbart, ${ }^{2}$ J. Strauss, ${ }^{2}$ W. Waltenberger, ${ }^{2}$ J. Wittmann, ${ }^{2}$ C.-E. Wulz, ${ }^{2, b}$ M. Zarucki, ${ }^{2}$ V. Chekhovsky, ${ }^{3}$ V. Mossolov, ${ }^{3}$ J. Suarez Gonzalez, ${ }^{3}$ E. A. De Wolf, ${ }^{4}$ D. Di Croce, ${ }^{4}$ X. Janssen, ${ }^{4}$ J. Lauwers, ${ }^{4}$ M. Van De Klundert, ${ }^{4}$ H. Van Haevermaet, ${ }^{4}$ P. Van Mechelen, ${ }^{4}$ N. Van Remortel, ${ }^{4}$ S. Abu Zeid, ${ }^{5}$ F. Blekman, ${ }^{5}$ J. D'Hondt, ${ }^{5}$

I. De Bruyn, ${ }^{5}$ J. De Clercq, ${ }^{5}$ K. Deroover, ${ }^{5}$ G. Flouris, ${ }^{5}$ D. Lontkovskyi, ${ }^{5}$ S. Lowette, ${ }^{5}$ S. Moortgat,${ }^{5}$ L. Moreels, ${ }^{5}$ A. Olbrechts, ${ }^{5}$ Q. Python, ${ }^{5}$ K. Skovpen, ${ }^{5}$ S. Tavernier, ${ }^{5}$ W. Van Doninck, ${ }^{5}$ P. Van Mulders, ${ }^{5}$ I. Van Parijs, ${ }^{5}$ H. Brun, ${ }^{6}$ B. Clerbaux, ${ }^{6}$ G. De Lentdecker, ${ }^{6}$ H. Delannoy, ${ }^{6}$ G. Fasanella, ${ }^{6}$ L. Favart, ${ }^{6}$ R. Goldouzian, ${ }^{6}$ A. Grebenyuk, ${ }_{6}^{6}$ G. Karapostoli, ${ }^{6}$

T. Lenzi, ${ }^{6}$ J. Luetic, ${ }^{6}$ T. Maerschalk, ${ }^{6}$ A. Marinov, ${ }^{6}$ A. Randle-conde, ${ }^{6}$ T. Seva, ${ }^{6}$ C. Vander Velde, ${ }^{6}$ P. Vanlaer, ${ }^{6}$

D. Vannerom ${ }^{6}$ R. Yonamine ${ }^{6}{ }^{\text {F. Zenoni, }}{ }^{6}$ F. Zhang, ${ }^{6, \mathrm{c}}$ A. Cimmino, ${ }^{7}$ T. Cornelis, ${ }^{7}$ D. Dobur, ${ }^{7}$ A. Fagot, ${ }^{7}$ M. Gul, ${ }^{7}$ I. Khvastunov, ${ }^{7}$ D. Poyraz, ${ }^{7}$ C. Roskas, ${ }^{7}$ S. Salva, ${ }^{7}$ M. Tytgat, ${ }^{7}$ W. Verbeke, ${ }^{7}$ N. Zaganidis, ${ }^{7}$ H. Bakhshiansohi, ${ }^{8}$ O. Bondu, ${ }^{8}$ S. Brochet, ${ }^{8}$ G. Bruno, ${ }^{8}$ A. Caudron, ${ }^{8}$ S. De Visscher, ${ }^{8}$ C. Delaere,${ }^{8}$ M. Delcourt ${ }^{8}$ B. Francois, ${ }^{8}$ A. Giammanco, ${ }^{8}$ A. Jafari, ${ }^{8}$ M. Komm, ${ }^{8}$ G. Krintiras, ${ }^{8}$ V. Lemaitre, ${ }^{8}$ A. Magitteri, ${ }^{8}$ A. Mertens, ${ }^{8}$ M. Musich,${ }^{8}$ K. Piotrzkowski, ${ }^{8}$ L. Quertenmont, ${ }^{8}$ M. Vidal Marono, ${ }^{8}$ S. Wertz, ${ }^{8}$ N. Beliy, ${ }^{9}$ W. L. Aldá Júnior, ${ }^{10}$ F. L. Alves,${ }^{10}$ G. A. Alves, ${ }^{10}$ L. Brito, ${ }^{10}$

M. Correa Martins Junior, ${ }^{10}$ C. Hensel,,${ }^{10}$ A. Moraes,${ }^{10}$ M. E. Pol, ${ }^{10}$ P. Rebello Teles, ${ }^{10}$ E. Belchior Batista Das Chagas,${ }^{11}$ W. Carvalho, ${ }^{11}$ J. Chinellato, ${ }^{11, d}$ A. Custódio, ${ }^{11}$ E. M. Da Costa, ${ }^{11}$ G. G. Da Silveira, ${ }^{11, e}$ D. De Jesus Damiao, ${ }^{11}$ S. Fonseca De Souza, ${ }^{11}$ L. M. Huertas Guativa, ${ }^{11}$ H. Malbouisson, ${ }^{11}$ M. Melo De Almeida, ${ }^{11}$ C. Mora Herrera, ${ }^{11}$ L. Mundim, ${ }^{11}$ H. Nogima, ${ }^{11}$ A. Santoro, ${ }^{11}$ A. Sznajder, ${ }^{11}$ E. J. Tonelli Manganote, ${ }^{11, d}$ F. Torres Da Silva De Araujo, ${ }^{11}$ A. Vilela Pereira, ${ }^{11}$ S. Ahuja, ${ }^{12 a}$ C. A. Bernardes, ${ }^{12 a}$ T. R. Fernandez Perez Tomei, ${ }^{12 a}$ E. M. Gregores,${ }^{12 b}$ P. G. Mercadante, ${ }^{12 b}$ S. F. Novaes, ${ }^{12 \mathrm{a}}$ Sandra S. Padula, ${ }^{12 \mathrm{a}}$ D. Romero Abad ${ }^{12 \mathrm{~b}}$ J. C. Ruiz Vargas, ${ }^{12 \mathrm{a}}$ A. Aleksandrov,${ }^{13}$ R. Hadjiiska, ${ }^{13}$ P. Iaydjiev, ${ }^{13}$ M. Misheva, ${ }^{13}$ M. Rodozov,${ }^{13}$ M. Shopova, ${ }^{13}$ S. Stoykova, ${ }^{13}$ G. Sultanov ${ }^{13}$ A. Dimitrov ${ }^{14}$ I. Glushkov, ${ }^{14}$ L. Litov ${ }^{14}$ B. Pavlov, ${ }^{14}$ P. Petkov ${ }^{14}$ W. Fang, ${ }^{15, f}$ X. Gao, ${ }^{15, f}$ M. Ahmad, ${ }^{16}$ J. G. Bian, ${ }^{16}$ G. M. Chen, ${ }^{16}$ H. S. Chen, ${ }^{16}$ M. Chen, ${ }^{16}$ Y. Chen, ${ }^{16}$ C. H. Jiang, ${ }^{16}$ D. Leggat,${ }^{16}$ Z. Liu, ${ }^{16}$ F. Romeo, ${ }^{16}$ S. M. Shaheen ${ }^{16}$ A. Spiezia, ${ }^{16}$ J. Tao, ${ }^{16}$ C. Wang,${ }^{16}$ Z. Wang, ${ }^{16}$ E. Yazgan, ${ }^{16}$ H. Zhang, ${ }^{16}$ J. Zhao, ${ }^{16}$ Y. Ban ${ }^{17}$ G. Chen, ${ }^{17}$ Q. Li, ${ }^{17}$ S. Liu, ${ }^{17}$ Y. Mao, ${ }^{17}$ S. J. Qian, ${ }^{17}$ D. Wang, ${ }^{17}$ Z. Xu, ${ }^{17}$ C. Avila, ${ }^{18}$ A. Cabrera, ${ }^{18}$ L. F. Chaparro Sierra, ${ }^{18}$ C. Florez, ${ }^{18}$ C. F. González Hernández, ${ }^{18}$ J. D. Ruiz Alvarez, ${ }^{18}$ B. Courbon, ${ }^{19}$ N. Godinovic, ${ }^{19}$ D. Lelas, ${ }^{19}$ I. Puljak, ${ }^{19}$ P. M. Ribeiro Cipriano, ${ }^{19}$ T. Sculac, ${ }^{19}$ Z. Antunovic, ${ }^{20}$ M. Kovac, ${ }^{20}$ V. Brigljevic, ${ }^{21}$ D. Ferencek, ${ }^{21}$ K. Kadija, ${ }^{21}$ B. Mesic, ${ }^{21}$ T. Susa, ${ }^{21}$ M. W. Ather, ${ }^{22}$ A. Attikis, ${ }^{22}$ G. Mavromanolakis, ${ }^{22}$ J. Mousa, ${ }^{22}$ C. Nicolaou, ${ }^{22}$ F. Ptochos, ${ }^{22}$ P. A. Razis,${ }^{22}$ H. Rykaczewski, ${ }^{22}$ M. Finger, ${ }^{23, g}$ M. Finger Jr., ${ }^{23, g}$ E. Carrera Jarrin, ${ }^{24}$ 
A. A. Abdelalim, ${ }^{25, h, i}$ Y. Mohammed ${ }^{25, j}$ E. Salama,${ }^{25, k, 1}$ R. K. Dewanjee, ${ }^{26}$ M. Kadastik, ${ }^{26}$ L. Perrini, ${ }^{26}$ M. Raidal, ${ }^{26}$ A. Tiko, ${ }^{26}$ C. Veelken, ${ }^{26}$ P. Eerola,${ }^{27}$ J. Pekkanen, ${ }^{27}$ M. Voutilainen, ${ }^{27}$ J. Härkönen, ${ }^{28}$ T. Järvinen, ${ }^{28}$ V. Karimäki, ${ }^{28}$ R. Kinnunen, ${ }^{28}$ T. Lampén, ${ }^{28} \mathrm{~K}$. Lassila-Perini, ${ }^{28} \mathrm{~S}$. Lehti, ${ }^{28} \mathrm{~T}$. Lindén, ${ }^{28} \mathrm{P}$. Luukka, ${ }^{28} \mathrm{E}$. Tuominen, ${ }^{28} \mathrm{~J}$. Tuominiemi, ${ }^{28}$ E. Tuovinen, ${ }^{28}$ J. Talvitie, ${ }^{29}$ T. Tuuva, ${ }^{29}$ M. Besancon,${ }^{30}$ F. Couderc,${ }^{30}$ M. Dejardin, ${ }^{30}$ D. Denegri, ${ }^{30}$ J. L. Faure,${ }^{30}$ F. Ferri, ${ }^{30}$ S. Ganjour, ${ }^{30}$ S. Ghosh ${ }^{30}$ A. Givernaud,${ }^{30}$ P. Gras, ${ }^{30}$ G. Hamel de Monchenault, ${ }^{30}$ P. Jarry, ${ }^{30}$ I. Kucher, ${ }^{30}$ E. Locci, ${ }^{30}$

M. Machet,${ }^{30}$ J. Malcles, ${ }^{30}$ G. Negro, ${ }^{30}$ J. Rander, ${ }^{30}$ A. Rosowsky, ${ }^{30}$ M. Ö. Sahin, ${ }^{30}$ M. Titov, ${ }^{30}$ A. Abdulsalam, ${ }^{31}$ I. Antropov, ${ }^{31}$ S. Baffioni, ${ }^{31}$ F. Beaudette, ${ }^{31}$ P. Busson, ${ }^{31}$ L. Cadamuro, ${ }^{31}$ C. Charlot, ${ }^{31}$ R. Granier de Cassagnac, ${ }^{31}$ M. Jo,${ }^{31}$ S. Lisniak, ${ }^{31}$ A. Lobanov,${ }^{31}$ J. Martin Blanco, ${ }^{31}$ M. Nguyen, ${ }^{31}$ C. Ochando, ${ }^{31}$ G. Ortona,${ }^{31}$ P. Paganini, ${ }^{31}$ P. Pigard,${ }^{31}$

S. Regnard, ${ }^{31}$ R. Salerno, ${ }^{31}$ J. B. Sauvan, ${ }^{31}$ Y. Sirois, ${ }^{31}$ A. G. Stahl Leiton, ${ }^{31}$ T. Strebler, ${ }^{31}$ Y. Yilmaz, ${ }^{31}$ A. Zabi, ${ }^{31}$ A. Zghiche, ${ }^{31}$ J.-L. Agram, ${ }^{32, \mathrm{~m}}$ J. Andrea, ${ }^{32}$ D. Bloch, ${ }^{32}$ J.-M. Brom, ${ }^{32}$ M. Buttignol, ${ }^{32}$ E. C. Chabert, ${ }^{32}$ N. Chanon, ${ }^{32}$ C. Collard ${ }^{32}$ E. Conte, ${ }^{32, \mathrm{~m}}$ X. Coubez, ${ }^{32}$ J.-C. Fontaine, ${ }^{32, \mathrm{~m}}$ D. Gelé, ${ }^{32}$ U. Goerlach, ${ }^{32}$ M. Jansová, ${ }^{32}$ A.-C. Le Bihan, ${ }^{32}$ N. Tonon, ${ }^{32}$ P. Van Hove, ${ }^{32}$ S. Gadrat,${ }^{33}$ S. Beauceron, ${ }^{34}$ C. Bernet, ${ }^{34}$ G. Boudoul,${ }^{34}$ R. Chierici, ${ }^{34}$ D. Contardo, ${ }^{34}$ P. Depasse, ${ }^{34}$ H. El Mamouni, ${ }^{34}$ J. Fay,${ }^{34}$ L. Finco, ${ }^{34}$ S. Gascon, ${ }^{34}$ M. Gouzevitch, ${ }^{34}$ G. Grenier,${ }^{34}$ B. Ille, ${ }^{34}$ F. Lagarde,${ }^{34}$ I. B. Laktineh, ${ }^{34}$ M. Lethuillier, ${ }^{34}$ L. Mirabito, ${ }^{34}$ A. L. Pequegnot, ${ }^{34}$ S. Perries,${ }^{34}$ A. Popov, ${ }^{34, n}$ V. Sordini,,${ }^{34}$ M. Vander Donckt, ${ }^{34}$ S. Viret, ${ }^{34}$ T. Toriashvili, ${ }^{35,0}$ D. Lomidze, ${ }^{36}$ C. Autermann, ${ }^{37}$ S. Beranek,${ }^{37}$ L. Feld, ${ }^{37}$ M. K. Kiesel, ${ }^{37}$ K. Klein, ${ }^{37}$ M. Lipinski, ${ }^{37}$ M. Preuten,${ }^{37}$ C. Schomakers, ${ }^{37}$ J. Schulz,${ }^{37}$ T. Verlage, ${ }^{37}$ A. Albert, ${ }^{38}$ M. Brodski, ${ }^{38}$

E. Dietz-Laursonn, ${ }^{38}$ D. Duchardt, ${ }^{38}$ M. Endres,${ }^{38}$ M. Erdmann, ${ }^{38}$ S. Erdweg, ${ }^{38}$ T. Esch, ${ }^{38}$ R. Fischer,${ }^{38}$ A. Güth, ${ }^{38}$ M. Hamer, ${ }^{38}$ T. Hebbeker, ${ }^{38}$ C. Heidemann, ${ }^{38}$ K. Hoepfner, ${ }^{38}$ S. Knutzen, ${ }^{38}$ M. Merschmeyer, ${ }^{38}$ A. Meyer, ${ }^{38}$ P. Millet, ${ }^{38}$ S. Mukherjee, ${ }^{38}$ M. Olschewski, ${ }^{38}$ K. Padeken, ${ }^{38}$ T. Pook, ${ }^{38}$ M. Radziej, ${ }^{38}$ H. Reithler, ${ }^{38}$ M. Rieger, ${ }^{38}$ F. Scheuch, ${ }^{38}$ D. Teyssier ${ }^{38}$ S. Thüer, ${ }^{38}$ G. Flügge, ${ }^{39}$ B. Kargoll, ${ }^{39}$ T. Kress, ${ }^{39}$ A. Künsken, ${ }^{39}$ J. Lingemann, ${ }^{39}$ T. Müller, ${ }^{39}$ A. Nehrkorn, ${ }^{39}$ A. Nowack, ${ }^{39}$ C. Pistone, ${ }^{39}$ O. Pooth,${ }^{39}$ A. Stahl, ${ }^{39, p}$ M. Aldaya Martin, ${ }^{40}$ T. Arndt,${ }^{40}$ C. Asawatangtrakuldee, ${ }^{40}$ K. Beernaert, ${ }^{40}$ O. Behnke, ${ }^{40}$ U. Behrens, ${ }^{40}$ A. Bermúdez Martínez ${ }^{40}$ A. A. Bin Anuar, ${ }^{40}$ K. Borras, ${ }^{40, q}$ V. Botta, ${ }^{40}$ A. Campbell, ${ }^{40}$ P. Connor, ${ }^{40}$ C. Contreras-Campana,${ }^{40}$ F. Costanza, ${ }^{40}$ C. Diez Pardos,${ }^{40}$ G. Eckerlin, ${ }^{40}$ D. Eckstein, ${ }^{40}$ T. Eichhorn, ${ }^{40}$ E. Eren, ${ }^{40}$ E. Gallo, ${ }^{40, r}$ J. Garay Garcia ${ }^{40}$ A. Geiser, ${ }^{40}$ A. Gizhko, ${ }^{40}$ J. M. Grados Luyando, ${ }^{40}$ A. Grohsjean, ${ }^{40}$ P. Gunnellini, ${ }^{40}$ A. Harb,${ }^{40}$ J. Hauk, ${ }^{40}$ M. Hempel,${ }^{40, s}$ H. Jung, ${ }^{40}$ A. Kalogeropoulos, ${ }^{40}$ M. Kasemann, ${ }^{40}$ J. Keaveney, ${ }^{40}$ C. Kleinwort, ${ }^{40}$ I. Korol, ${ }^{40}$ D. Krücker, ${ }^{40}$ W. Lange, ${ }^{40}$ A. Lelek, ${ }^{40}$ T. Lenz,${ }^{40}$ J. Leonard, ${ }^{40}$ K. Lipka, ${ }^{40}$ W. Lohmann, ${ }^{40, s}$ R. Mankel, ${ }^{40}$ I.-A. Melzer-Pellmann, ${ }^{40}$ A. B. Meyer, ${ }^{40}$ G. Mittag, ${ }^{40}$ J. Mnich, ${ }^{40}$ A. Mussgiller, ${ }^{40}$ E. Ntomari, ${ }^{40}$ D. Pitzl, ${ }^{40}$ R. Placakyte, ${ }^{40}$ A. Raspereza,${ }^{40}$ B. Roland,${ }^{40}$ M. Savitskyi ${ }^{40}$ P. Saxena,${ }^{40}$ R. Shevchenko, ${ }^{40}$ S. Spannagel,${ }^{40}$ N. Stefaniuk,${ }^{40}$ G. P. Van Onsem, ${ }^{40}$ R. Walsh, ${ }^{40}$ Y. Wen, ${ }^{40}$ K. Wichmann, ${ }^{40}$ C. Wissing, ${ }^{40}$ O. Zenaiev, ${ }^{40}$ S. Bein, ${ }^{41}$ V. Blobel,,${ }^{41}$ M. Centis Vignali, ${ }^{41}$ A. R. Draeger, ${ }^{41}$ T. Dreyer, ${ }^{41}$ E. Garutti, ${ }^{41}$ D. Gonzalez, ${ }^{41}$ J. Haller, ${ }^{41}$ A. Hinzmann, ${ }^{41}$ M. Hoffmann,${ }^{41}$ A. Karavdina, ${ }^{41}$ R. Klanner, ${ }^{41}$ R. Kogler, ${ }^{41}$ N. Kovalchuk, ${ }^{41}$ S. Kurz, ${ }^{41}$ T. Lapsien, ${ }^{41}$ I. Marchesini, ${ }^{41}$ D. Marconi, ${ }^{41}$ M. Meyer, ${ }^{41}$ M. Niedziela, ${ }^{41}$ D. Nowatschin ${ }^{41}$ F. Pantaleo, ${ }^{41, p}$ T. Peiffer, ${ }^{41}$ A. Perieanu, ${ }^{41}$ C. Scharf, ${ }^{41}$ P. Schleper, ${ }^{41}$ A. Schmidt, ${ }^{41}$ S. Schumann, ${ }^{41}$ J. Schwandt, ${ }^{41}$ J. Sonneveld, ${ }^{41}$ H. Stadie, ${ }^{41}$ G. Steinbrück, ${ }^{41}$ F. M. Stober, ${ }^{41}$ M. Stöver ${ }^{41}$ H. Tholen, ${ }^{41}$ D. Troendle, ${ }^{41}$ E. Usai, ${ }^{41}$ L. Vanelderen ${ }^{41}$ A. Vanhoefer, ${ }^{41}$ B. Vormwald, ${ }^{41}$ M. Akbiyik, ${ }^{42}$ C. Barth, ${ }^{42}$ S. Baur, ${ }^{42}$ E. Butz ${ }^{42}$ R. Caspart, ${ }^{42}$ T. Chwalek, ${ }^{42}$ F. Colombo, ${ }^{42}$ W. De Boer, ${ }^{42}$ A. Dierlamm, ${ }^{42}$ B. Freund, ${ }^{42}$ R. Friese, ${ }^{42}$ M. Giffels ${ }^{42}$ A. Gilbert, ${ }^{42}$ D. Haitz, ${ }^{42}$ F. Hartmann, ${ }^{42, p}$ S. M. Heindl, ${ }^{42}$ U. Husemann, ${ }^{42}$ F. Kassel, ${ }^{42, p}$ S. Kudella, ${ }^{42}$ H. Mildner, ${ }^{42}$ M. U. Mozer, ${ }^{42}$ Th. Müller ${ }^{42}$ M. Plagge, ${ }^{42}$ G. Quast ${ }^{42}$ K. Rabbertz, ${ }^{42}$ M. Schröder, ${ }^{42}$ I. Shvetsov, ${ }^{42}$ G. Sieber, ${ }^{42}$ H. J. Simonis, ${ }^{42}$ R. Ulrich, ${ }^{42}$ S. Wayand, ${ }^{42}$ M. Weber, ${ }^{42}$ T. Weiler, ${ }^{42}$ S. Williamson, ${ }^{42}$ C. Wöhrmann, ${ }^{42}$ R. Wolf, ${ }^{42}$ G. Anagnostou, ${ }^{43}$ G. Daskalakis, ${ }^{43}$ T. Geralis, ${ }^{43}$ V. A. Giakoumopoulou, ${ }^{43}$ A. Kyriakis, ${ }^{43}$ D. Loukas, ${ }^{43}$ I. Topsis-Giotis ${ }^{43}$ S. Kesisoglou, ${ }^{44}$ A. Panagiotou, ${ }^{44}$ N. Saoulidou, ${ }^{44}$ I. Evangelou, ${ }^{45}$ C. Foudas, ${ }^{45}$ P. Kokkas, ${ }^{45}$ N. Manthos, ${ }^{45}$ I. Papadopoulos, ${ }^{45}$ E. Paradas, ${ }^{45}$ J. Strologas, ${ }^{45}$ F. A. Triantis, ${ }^{45}$ M. Csanad, ${ }^{46}$ N. Filipovic, ${ }^{46}$ G. Pasztor,${ }^{46}$ G. Bencze, ${ }^{47}$ C. Hajdu, ${ }^{47}$ D. Horvath, ${ }^{47, t}$ Á. Hunyadi, ${ }^{47}$ F. Sikler,${ }^{47}$ V. Veszpremi,${ }^{47}$ G. Vesztergombi, ${ }^{47, u}$ A. J. Zsigmond, ${ }^{47}$ N. Beni, ${ }^{48}$ S. Czellar, ${ }^{48}$ J. Karancsi, ${ }^{48, v}$ A. Makovec, ${ }^{48}$ J. Molnar, ${ }^{48}$ Z. Szillasi, ${ }^{48}$ M. Bartók, ${ }^{49, u}$ P. Raics, ${ }^{49}$ Z. L. Trocsanyi, ${ }^{49}$ B. Ujvari, ${ }^{49}$ S. Choudhury, ${ }^{50}$ J. R. Komaragiri, ${ }^{50}$ S. Bahinipati, ${ }^{51, w}$ S. Bhowmik, ${ }^{51}$ P. Mal, ${ }^{51}$ K. Mandal,${ }^{51}$ A. Nayak, ${ }^{51, x}$ D. K. Sahoo, ${ }^{51, w}$ N. Sahoo, ${ }^{51}$ S. K. Swain, ${ }^{51}$ S. Bansal,${ }^{52}$ S. B. Beri, ${ }^{52}$ V. Bhatnagar, ${ }^{52}$ U. Bhawandeep, ${ }^{52}$ R. Chawla, ${ }^{52}$ N. Dhingra, ${ }^{52}$ A. K. Kalsi, ${ }^{52}$ A. Kaur, ${ }^{52}$ M. Kaur, ${ }^{52}$ R. Kumar, ${ }^{52}$ P. Kumari, ${ }^{52}$ A. Mehta, ${ }^{52}$ J. B. Singh, ${ }^{52}$ G. Walia, ${ }^{52}$ Ashok Kumar, ${ }^{53}$ Aashaq Shah, ${ }^{53}$ A. Bhardwaj, ${ }^{53}$ S. Chauhan, ${ }^{53}$ B. C. Choudhary, ${ }^{53}$ R. B. Garg, ${ }^{53}$ S. Keshri, ${ }^{53}$ A. Kumar, ${ }^{53}$ S. Malhotra, ${ }^{53}$ M. Naimuddin, ${ }^{53}$ K. Ranjan, ${ }^{53}$ R. Sharma, ${ }^{53}$ V. Sharma,${ }^{53}$ R. Bhardwaj, ${ }^{54}$ R. Bhattacharya, ${ }^{54}$ S. Bhattacharya, ${ }^{54}$ S. Dey, ${ }^{54}$ S. Dutt, ${ }^{54}$ S. Dutta, ${ }^{54}$ S. Ghosh, ${ }^{54}$ N. Majumdar, ${ }^{54}$ A. Modak, ${ }^{54}$ K. Mondal,,${ }^{54}$ 
S. Mukhopadhyay, ${ }^{54}$ S. Nandan, ${ }^{54}$ A. Purohit, ${ }^{54}$ A. Roy ${ }^{54}$ D. Roy, ${ }^{54}$ S. Roy Chowdhury, ${ }^{54}$ S. Sarkar, ${ }^{54}$ M. Sharan,${ }^{54}$ S. Thakur, ${ }^{54}$ P. K. Behera, ${ }^{55}$ R. Chudasama, ${ }^{56}$ D. Dutta, ${ }^{56}$ V. Jha, ${ }^{56}$ V. Kumar, ${ }^{56}$ A. K. Mohanty, ${ }^{56, p}$ P. K. Netrakanti, ${ }^{56}$ L. M. Pant ${ }^{56}$ P. Shukla, ${ }^{56}$ A. Topkar, ${ }^{56}$ T. Aziz,${ }^{57}$ S. Dugad,${ }^{57}$ B. Mahakud,${ }^{57}$ S. Mitra,${ }^{57}$ G. B. Mohanty, ${ }^{57}$ B. Parida,${ }^{57}$ N. Sur, ${ }^{57}$ B. Sutar, ${ }^{57}$ S. Banerjee, ${ }^{58}$ S. Bhattacharya, ${ }^{58}$ S. Chatterjee, ${ }^{58}$ P. Das, ${ }^{58}$ M. Guchait, ${ }^{58}$ Sa. Jain, ${ }^{58}$ S. Kumar,${ }^{58}$ M. Maity, ${ }^{58, y}$ G. Majumder, ${ }^{58}$ K. Mazumdar, ${ }^{58}$ T. Sarkar, ${ }^{58, y}$ N. Wickramage, ${ }^{58, z}$ S. Chauhan,${ }^{59}$ S. Dube, ${ }^{59}$ V. Hegde, ${ }^{59}$

A. Kapoor, ${ }^{59}$ K. Kothekar, ${ }^{59}$ S. Pandey, ${ }^{59}$ A. Rane, ${ }^{59}$ S. Sharma, ${ }^{59}$ S. Chenarani, ${ }^{60, \text { aa }}$ E. Eskandari Tadavani, ${ }^{60}$ S. M. Etesami, ${ }^{60, a a}$ M. Khakzad,${ }^{60}$ M. Mohammadi Najafabadi, ${ }^{60}$ M. Naseri, ${ }^{60}$ S. Paktinat Mehdiabadi, ${ }^{60, b b}$ F. Rezaei Hosseinabadi, ${ }^{60}$ B. Safarzadeh, ${ }^{60, c c}$ M. Zeinali, ${ }^{60}$ M. Felcini, ${ }^{61}$ M. Grunewald, ${ }^{61}$ M. Abbrescia,${ }^{62 a, 62 b}$ C. Calabria, ${ }^{62 a, 62 b}$ C. Caputo, ${ }^{62 a, 62 b}$ A. Colaleo, ${ }^{62 a}$ D. Creanza, ${ }^{62 a, 62 c}$ L. Cristella, ${ }^{62 a, 62 b}$ N. De Filippis, ${ }^{62 a, 62 c}$ M. De Palma, ${ }^{62 a, 62 b}$ F. Errico, ${ }^{62 a, 62 b}$ L. Fiore, ${ }^{62 a}$ G. Iaselli, ${ }^{62 a, 62 c}$ S. Lezki, ${ }^{62 a, 62 b}$ G. Maggi, ${ }^{62 a, 62 c}$ M. Maggi, ${ }^{62 a}$ G. Miniello, ${ }^{62 a, 62 b}$ S. My, ${ }^{62 a, 62 b}$ S. Nuzzo, ${ }^{62 a, 62 b}$ A. Pompili, ${ }^{62 a, 62 b}$ G. Pugliese, ${ }^{62 a, 62 c}$ R. Radogna, ${ }^{62 a, 62 b}$ A. Ranieri, ${ }^{62 a}$ G. Selvaggi, ${ }^{62 a, 62 b}$ A. Sharma, ${ }^{62 a}$ L. Silvestris, ${ }^{62 a, p}$ R. Venditti, ${ }^{62 a}$ P. Verwilligen, ${ }^{62 a}$ G. Abbiendi, ${ }^{63 a}$ C. Battilana ${ }^{63 a, 63 b}$ D. Bonacorsi,${ }^{63 a, 63 b}$ S. Braibant-Giacomelli, ${ }^{63 a, 63 b}$ L. Brigliadori, ${ }^{63 a, 63 b}$ R. Campanini, ${ }^{63 a, 63 b}$ P. Capiluppi, ${ }^{63 a, 63 b}$ A. Castro, ${ }^{63 a, 63 b}$ F. R. Cavallo, ${ }^{63 a}$ S. S. Chhibra, ${ }^{63 a}$ G. Codispoti, ${ }^{63 a, 63 b}$ M. Cuffiani, ${ }^{63 a, 63 b}$ G. M. Dallavalle, ${ }^{63 a}$ F. Fabbri, ${ }^{63 a}$ A. Fanfani, ${ }^{63 a, 63 b}$ D. Fasanella, ${ }^{63 a, 63 b}$ P. Giacomelli, ${ }^{63 a}$ L. Guiducci, ${ }^{63 a, 63 b}$ S. Marcellini, ${ }^{63 a}$ G. Masetti, ${ }^{63 a}$ F. L. Navarria, ${ }^{63 a, 63 b}$ A. Perrotta, ${ }^{63 a}$ A. M. Rossi, ${ }^{63 a, 63 b}$ T. Rovelli, ${ }^{63 a, 63 b}$ G. P. Siroli ${ }^{63 a, 63 b}$ N. Tosi,${ }^{63 a}$ S. Albergo,${ }^{64 a, 64 b}$ S. Costa, ${ }^{64 a, 64 b}$

A. Di Mattia, ${ }^{64 a}$ F. Giordano, ${ }^{64 a, 64 b}$ R. Potenza ${ }^{64 a, 64 b}$ A. Tricomi,${ }^{64 a, 64 b}$ C. Tuve ${ }^{64 a, 64 b}$ G. Barbagli, ${ }^{65 a}$ K. Chatterjee ${ }^{65 a, 65 b}$ V. Ciulli ${ }^{65 a, 65 b}$ C. Civinini, ${ }^{65 a}$ R. D’Alessandro, ${ }^{65 a, 65 b}$ E. Focardi, ${ }^{65 a, 65 b}$ P. Lenzi, ${ }^{65 a, 65 b}$ M. Meschini, ${ }^{65 a}$ S. Paoletti ${ }^{65 a}$ L. Russo, ${ }^{65 a, d d}$ G. Sguazzoni, ${ }^{65 a}$ L. Viliani, ${ }^{65 a, 65 b, p}$ L. Benussi ${ }^{66}$ S. Bianco, ${ }^{66}$ F. Fabbri, ${ }^{66}$ D. Piccolo, ${ }^{66}$ F. Primavera, ${ }^{66, p}$ V. Calvelli, ${ }^{67 a, 67 b}$ F. Ferro, ${ }^{67 a}$ E. Robutti, ${ }^{67 a}$ S. Tosi, ${ }^{67 a, 67 b}$ L. Brianza,${ }^{68 a, 68 b}$ F. Brivio, ${ }^{68 a, 68 b}$ V. Ciriolo, ${ }^{68 a, 68 b}$ M. E. Dinardo, ${ }^{68 a, 68 b}$ S. Fiorendi, ${ }^{68,68 b}$ S. Gennai, ${ }^{68 a}$ A. Ghezzi, ${ }^{68,68 b}$ P. Govoni, ${ }^{68 a, 68 b}$ M. Malberti, ${ }^{68 a, 68 b}$ S. Malvezzi, ${ }^{68 a}$ R. A. Manzoni, ${ }^{68 a, 68 b}$ D. Menasce, ${ }^{68 \mathrm{a}}$ L. Moroni, ${ }^{68 \mathrm{a}}$ M. Paganoni, ${ }^{68 \mathrm{a}, 68 \mathrm{~b}}$ K. Pauwels, ${ }^{68 \mathrm{a}, 68 \mathrm{~b}}$ D. Pedrini, ${ }^{68 \mathrm{a}}$ S. Pigazzini, ${ }^{68,68 \mathrm{~b}, \text { ee }}$ S. Ragazzi, ${ }^{68 a, 68 b}$ T. Tabarelli de Fatis, ${ }^{68 a, 68 b}$ S. Buontempo ${ }^{69 a}$ N. Cavallo, ${ }^{69 a, 69 c}$ S. Di Guida,${ }^{69 a, 69 d, p}$ F. Fabozzi ${ }^{69 a, 69 c}$ F. Fienga ${ }^{69 a, 69 b}$ A. O. M. Iorio, ${ }^{69 a, 69 b}$ W. A. Khan, ${ }^{69 a}$ L. Lista ${ }^{69 a}$ S. Meola, ${ }^{69 a, 69 d, p}$ P. Paolucci, ${ }^{69 a, p}$ C. Sciacca,${ }^{69 a, 69 b}$ F. Thyssen, ${ }^{69 a}$ P. Azzi, ${ }^{70 a, p}$ N. Bacchetta, ${ }^{70 a}$ L. Benato,${ }^{70 a, 70 b}$ D. Bisello, ${ }^{70 a, 70 b}$ A. Boletti, ${ }^{70 a, 70 b}$ R. Carlin, ${ }^{70 a, 70 b}$ A. Carvalho Antunes De Oliveira, ${ }^{70 a, 70 b}$ P. Checchia, ${ }^{70 a}$ P. De Castro Manzano, ${ }^{70 a}$ T. Dorigo, ${ }^{70 a}$ U. Dosselli, ${ }^{70 a}$ F. Gasparini, ${ }^{70 a, 70 b}$ U. Gasparini, ${ }^{70 a, 70 b}$ A. Gozzelino, ${ }^{70 a}$ S. Lacaprara, ${ }^{70 a}$ M. Margoni, ${ }^{70 a, 70 b}$ A. T. Meneguzzo, ${ }^{70 a, 70 b}$ N. Pozzobon, ${ }^{70 a, 70 b}$ P. Ronchese, ${ }^{70 a, 70 b}$ R. Rossin,${ }^{70 a, 70 b}$ F. Simonetto, ${ }^{70 a, 70 b}$ E. Torassa, ${ }^{70 a}$ M. Zanetti, ${ }^{70 a, 70 b}$ P. Zotto, ${ }^{70 a, 70 b}$ G. Zumerle, ${ }^{70 a, 70 b}$ A. Braghieri, ${ }^{71 a}$ F. Fallavollita, ${ }^{71 a, 71 b}$ A. Magnani, ${ }^{71 a, 71 b}$ P. Montagna, ${ }^{71 a, 71 b}$ S. P. Ratti, ${ }^{71 a, 71 b}$ V. Re ${ }^{71 a}$ M. Ressegotti, ${ }^{71 \mathrm{a}}$ C. Riccardi, ${ }^{71 \mathrm{a}, 71 \mathrm{~b}}$ P. Salvini, ${ }^{71 \mathrm{a}}$ I. Vai, ${ }^{71 \mathrm{a}, 71 \mathrm{~b}}$ P. Vitulo, ${ }^{71 \mathrm{a}, 71 \mathrm{~b}}$ L. Alunni Solestizi, ${ }^{72 \mathrm{a}, 72 \mathrm{~b}}$ M. Biasini, ${ }^{72 \mathrm{a}, 72 \mathrm{~b}}$ G. M. Bilei, ${ }^{72 a}$ C. Cecchi, ${ }^{72 a}$ D. Ciangottini, ${ }^{72 a, 72 b}$ L. Fanò, ${ }^{72 a, 72 b}$ P. Lariccia, ${ }^{72 a, 72 b}$ R. Leonardi, ${ }^{72 a, 72 b}$ E. Manoni, ${ }^{72 a}$ G. Mantovani, ${ }^{72 a, 72 b}$ V. Mariani, ${ }^{72 a, 72 b}$ M. Menichelli, ${ }^{72 a}$ A. Rossi ${ }^{72 a}$ A. Saha, ${ }^{72 a}$ A. Santocchia, ${ }^{72 a, 72 b}$ D. Spiga, ${ }^{72 a}$

K. Androsov, ${ }^{73 a}$ P. Azzurri, ${ }^{73 a, p}$ G. Bagliesi, ${ }^{73 a}$ J. Bernardini, ${ }^{73 a}$ T. Boccali, ${ }^{73 a}$ L. Borrello, ${ }^{73 a}$ R. Castaldi, ${ }^{73 a}$ M. A. Ciocci, ${ }^{73 a, 73 b}$ R. Dell'Orso, ${ }^{73 a}$ G. Fedi, ${ }^{73 a}$ L. Giannini, ${ }^{73 a, 73 c}$ A. Giassi, ${ }^{73 a}$ M. T. Grippo, ${ }^{73 a, d d}$ F. Ligabue, ${ }^{73 a, 73 c}$ T. Lomtadze, ${ }^{73 a}$ E. Manca, ${ }^{73 a, 73 c}$ G. Mandorli, ${ }^{73 a, 73 c}$ L. Martini, ${ }^{73 a, 73 b}$ A. Messineo, ${ }^{73 a, 73 b}$ F. Palla, ${ }^{73 a}$ A. Rizzi, ${ }^{73 a, 73 b}$ A. Savoy-Navarro, ${ }^{73 a, f f}$ P. Spagnolo, ${ }^{73 a}$ R. Tenchini, ${ }^{73 a}$ G. Tonelli, ${ }^{73 a, 73 b}$ A. Venturi, ${ }^{73 a}$ P. G. Verdini, ${ }^{73 a}$ L. Barone, ${ }^{74 a, 74 b}$ F. Cavallari, ${ }^{74 a}$ M. Cipriani, ${ }^{74 a, 74 b}$ D. Del Re ${ }^{74 a, 74 b, p}$ M. Diemoz,${ }^{74 a}$ S. Gelli, ${ }^{74 a, 74 b}$ E. Longo, ${ }^{74 a, 74 b}$ F. Margaroli, ${ }^{74 a, 74 b}$ B. Marzocchi, ${ }^{74,74 b}$ P. Meridiani, ${ }^{74 a}$ G. Organtini, ${ }^{74 a, 74 b}$ R. Paramatti, ${ }^{74 a, 74 b}$ F. Preiato, ${ }^{74 a, 74 b}$ S. Rahatlou, ${ }^{74 a, 74 b}$ C. Rovelli, ${ }^{74 a}$ F. Santanastasio, ${ }^{74 a, 74 b}$ N. Amapane, ${ }^{75 a, 75 b}$ R. Arcidiacono, ${ }^{75 a, 75 c}$ S. Argiro, ${ }^{75 a, 75 b}$ M. Arneodo, ${ }^{75 a, 75 c}$ N. Bartosik, ${ }^{75 a}$ R. Bellan, ${ }^{75 a, 75 b}$ C. Biino, ${ }^{75 a}$ N. Cartiglia, ${ }^{75 a}$ F. Cenna, ${ }^{75 a, 75 b}$ M. Costa, ${ }^{75 a, 75 b}$ R. Covarelli, ${ }^{75 a, 75 b}$ A. Degano, ${ }^{75 a, 75 b}$ N. Demaria, ${ }^{75 a}$ B. Kiani, ${ }^{75 a, 75 b}$ C. Mariotti, ${ }^{75 a}$ S. Maselli, ${ }^{75 a}$ E. Migliore, ${ }^{75 a, 75 b}$ V. Monaco, ${ }^{75 a, 75 b}$ E. Monteil, ${ }^{75 a, 75 b}$ M. Monteno, ${ }^{75 a}$ M. M. Obertino, ${ }^{75 a, 75 b}$ L. Pacher, ${ }^{75 a, 75 b}$ N. Pastrone, ${ }^{75 a}$ M. Pelliccioni, ${ }^{75 a}$ G. L. Pinna Angioni, ${ }^{75 a, 75 b}$ F. Ravera ${ }^{75 a, 75 b}$ A. Romero, ${ }^{75 a, 75 b}$ M. Ruspa, ${ }^{75 a, 75 c}$ R. Sacchi, ${ }^{75 a, 75 b}$ K. Shchelina, ${ }^{75 a, 75 b}$ V. Sola, ${ }^{75 a}$ A. Solano, ${ }^{75 a, 75 b}$ A. Staiano, ${ }^{75 a}$ P. Traczyk,${ }^{75 a, 75 b}$ S. Belforte ${ }^{76 a}$ M. Casarsa ${ }^{76 a}$ F. Cossutti ${ }^{76 a}$ G. Della Ricca, ${ }^{76 a, 76 b}$ A. Zanetti, ${ }^{76 a}$ D. H. Kim, ${ }^{77}$ G. N. Kim, ${ }^{77}$ M. S. Kim,${ }^{77}$ J. Lee, ${ }^{77}$ S. Lee, ${ }^{77}$ S. W. Lee,${ }^{77}$ C. S. Moon,${ }^{77}$ Y. D. Oh,${ }^{77}$ S. Sekmen,${ }^{77}$ D. C. Son, ${ }^{77}$ Y. C. Yang,${ }^{77}$ A. Lee, ${ }^{78}$ H. Kim, ${ }^{79}$ D. H. Moon, ${ }^{79}$ G. Oh, ${ }^{79}$ J. A. Brochero Cifuentes, ${ }^{80}$ J. Goh,${ }^{80}$ T. J. Kim, ${ }^{80}$ S. Cho, ${ }^{81}$ S. Choi, ${ }^{81}$ Y. Go,${ }^{81}$ D. Gyun, ${ }^{81}$ S. Ha, ${ }^{81}$ B. Hong, ${ }^{81}$ Y. Jo, ${ }^{81}$ Y. Kim ${ }^{81}$ K. Lee, ${ }^{81}$ K. S. Lee, ${ }^{81}$ S. Lee, ${ }^{81}$ J. Lim, ${ }^{81}$ S. K. Park, ${ }^{81}$ Y. Roh, ${ }^{81}$ J. Almond ${ }^{82}$ J. Kim, ${ }^{82}$ J. S. Kim, ${ }^{82}$ H. Lee, ${ }^{82}$ K. Lee, ${ }^{82}$ K. Nam, ${ }^{82}$ S. B. Oh ${ }^{82}$ B. C. Radburn-Smith, ${ }^{82}$ S. h. Seo, ${ }^{82}$ U. K. Yang, ${ }^{82}$ H. D. Yoo, ${ }^{82}$ G. B. Yu, ${ }^{82}$ M. Choi,${ }^{83}$ H. Kim, ${ }^{83}$ J. H. Kim, ${ }^{83}$ J. S. H. Lee, ${ }^{83}$ I. C. Park, ${ }^{83}$ G. Ryu ${ }^{83}$ Y. Choi, ${ }^{84}$ 
C. Hwang, ${ }^{84}$ J. Lee, ${ }^{84}$ I. Yu, ${ }^{84}$ V. Dudenas, ${ }^{85}$ A. Juodagalvis, ${ }^{85}$ J. Vaitkus, ${ }^{85}$ I. Ahmed, ${ }^{86}$ Z. A. Ibrahim, ${ }^{86}$ M. A. B. Md Ali, ${ }^{86, g g}$ F. Mohamad Idris, ${ }^{86, \text { hh }}$ W. A. T. Wan Abdullah, ${ }^{86}$ M. N. Yusli, ${ }^{86}$ Z. Zolkapli, ${ }^{86}$ H. Castilla-Valdez, ${ }^{87}$ E. De La Cruz-Burelo, ${ }^{87}$ I. Heredia-De La Cruz, ${ }^{87, i i}$ R. Lopez-Fernandez, ${ }^{87}$ J. Mejia Guisao, ${ }^{87}$ A. Sanchez-Hernandez, ${ }^{87}$ S. Carrillo Moreno, ${ }^{88}$ C. Oropeza Barrera ${ }^{88}$ F. Vazquez Valencia, ${ }^{88}$ I. Pedraza ${ }^{89}$ H. A. Salazar Ibarguen, ${ }^{89}$ C. Uribe Estrada, ${ }^{89}$ A. Morelos Pineda, ${ }^{90}$ D. Krofcheck, ${ }^{91}$ P. H. Butler, ${ }^{92}$ A. Ahmad, ${ }^{93}$ M. Ahmad, ${ }^{93}$ Q. Hassan, ${ }^{93}$ H. R. Hoorani, ${ }^{93}$ A. Saddique, ${ }^{93}$ M. A. Shah, ${ }^{93}$ M. Shoaib,${ }^{93}$ M. Waqas, ${ }^{93}$ H. Bialkowska, ${ }^{94}$ M. Bluj,${ }^{94}$ B. Boimska, ${ }^{94}$ T. Frueboes,${ }^{94}$ M. Górski ${ }^{94}$ M. Kazana,${ }^{94}$ K. Nawrocki,${ }^{94}$ K. Romanowska-Rybinska, ${ }^{94}$ M. Szleper, ${ }^{94}$ P. Zalewski, ${ }^{94}$ K. Bunkowski, ${ }^{95}$ A. Byszuk, ${ }^{95, j j}$ K. Doroba, ${ }^{95}$ A. Kalinowski, ${ }^{95}$ M. Konecki, ${ }^{95}$ J. Krolikowski, ${ }^{95}$ M. Misiura, ${ }^{95}$ M. Olszewski, ${ }^{95}$ A. Pyskir, ${ }^{95}$ M. Walczak, ${ }^{95}$ P. Bargassa ${ }^{96}$ C. Beirão Da Cruz E Silva, ${ }^{96}$ B. Calpas, ${ }^{96}$ A. Di Francesco, ${ }^{96}$ P. Faccioli, ${ }^{96}$ M. Gallinaro, ${ }^{96}$ J. Hollar,${ }^{96}$ N. Leonardo, ${ }^{96}$ L. Lloret Iglesias, ${ }^{96}$ M. V. Nemallapudi, ${ }^{96}$ J. Seixas,${ }^{96}$ O. Toldaiev,${ }^{96}$ D. Vadruccio, ${ }^{96}$ J. Varela, ${ }^{96}$ S. Afanasiev, ${ }^{97}$ P. Bunin, ${ }^{97}$ M. Gavrilenko, ${ }^{97}$ I. Golutvin, ${ }^{97}$ I. Gorbunov, ${ }^{97}$ A. Kamenev, ${ }^{97}$ V. Karjavin, ${ }^{97}$ A. Lanev,${ }^{97}$ A. Malakhov, ${ }^{97}$ V. Matveev, ${ }^{97, k k, 11}$ V. Palichik,${ }^{97}$ V. Perelygin, ${ }^{97}$ S. Shmatov, ${ }^{97}$ S. Shulha, ${ }^{97}$ N. Skatchkov, ${ }^{97}$ V. Smirnov, ${ }^{97}$ N. Voytishin, ${ }^{97}$ A. Zarubin, ${ }^{97}$ Y. Ivanov, ${ }^{98}$ V. Kim, ${ }^{98, m m}$ E. Kuznetsova, ${ }^{98, n n}$ P. Levchenko, ${ }^{98}$ V. Murzin, ${ }^{98}$ V. Oreshkin, ${ }^{98}$ I. Smirnov, ${ }^{98}$ V. Sulimov, ${ }^{98}$ L. Uvarov, ${ }^{98}$ S. Vavilov, ${ }^{98}$ A. Vorobyev, ${ }^{98}$ Yu. Andreev, ${ }^{99}$ A. Dermenev, ${ }^{99}$ S. Gninenko, ${ }^{99}$ N. Golubev, ${ }^{99}$ A. Karneyeu, ${ }^{99}$ M. Kirsanov, ${ }^{99}$ N. Krasnikov, ${ }^{99}$ A. Pashenkov, ${ }^{99}$ D. Tlisov, ${ }^{99}$ A. Toropin, ${ }^{99}$ V. Epshteyn, ${ }^{100}$ V. Gavrilov, ${ }^{100}$ N. Lychkovskaya, ${ }^{100}$ V. Popov, ${ }^{100}$ I. Pozdnyakov, ${ }^{100}$ G. Safronov, ${ }^{100}$ A. Spiridonov, ${ }^{100}$ A. Stepennov, ${ }^{100}$ M. Toms, ${ }^{100}$ E. Vlasov, ${ }^{100}$ A. Zhokin, ${ }^{100}$ T. Aushev, ${ }^{101}$ A. Bylinkin, ${ }^{101,11}$ R. Chistov, ${ }^{102,00}$ M. Danilov, ${ }^{102,00}$ P. Parygin, ${ }^{102}$ D. Philippov, ${ }^{102}$ S. Polikarpov, ${ }^{102}$ E. Tarkovskii, ${ }^{102}$ V. Andreev, ${ }^{103}$ M. Azarkin, ${ }^{103,11}$

I. Dremin, ${ }^{103,11}$ M. Kirakosyan, ${ }^{103,11}$ A. Terkulov, ${ }^{103}$ A. Baskakov, ${ }^{104}$ A. Belyaev, ${ }^{104}$ E. Boos, ${ }^{104}$ V. Bunichev, ${ }^{104}$ M. Dubinin, ${ }^{104, p p}$ L. Dudko, ${ }^{104}$ A. Ershov, ${ }^{104}$ A. Gribushin, ${ }^{104}$ V. Klyukhin, ${ }^{104}$ O. Kodolova, ${ }^{104}$ I. Lokhtin, ${ }^{104}$ I. Miagkov, ${ }^{104}$ S. Obraztsov, ${ }^{104}$ S. Petrushanko, ${ }^{104}$ V. Savrin, ${ }^{104}$ V. Blinov, ${ }^{105, q q}$ Y. Skovpen, ${ }^{105, q q}$ D. Shtol, ${ }^{105, q q}$ I. Azhgirey, ${ }^{106}$ I. Bayshev, ${ }^{106}$ S. Bitioukov, ${ }^{106}$ D. Elumakhov, ${ }^{106}$ V. Kachanov ${ }^{106}$ A. Kalinin,${ }^{106}$ D. Konstantinov, ${ }^{106}$ V. Krychkine, ${ }^{106}$ V. Petrov ${ }^{106}$ R. Ryutin, ${ }^{106}$ A. Sobol, ${ }^{106}$ S. Troshin, ${ }^{106}$ N. Tyurin, ${ }^{106}$ A. Uzunian, ${ }^{106}$ A. Volkov, ${ }^{106}$ P. Adzic, ${ }^{107, \text { rr }}$ P. Cirkovic, ${ }^{107}$ D. Devetak, ${ }^{107}$ M. Dordevic, ${ }^{107}$ J. Milosevic, ${ }^{107}$ V. Rekovic, ${ }^{107}$ J. Alcaraz Maestre, ${ }^{108}$ M. Barrio Luna, ${ }^{108}$ M. Cerrada, ${ }^{108}$ N. Colino, ${ }^{108}$ B. De La Cruz, ${ }^{108}$ A. Delgado Peris, ${ }^{108}$ A. Escalante Del Valle, ${ }^{108}$ C. Fernandez Bedoya,${ }^{108}$ J. P. Fernández Ramos, ${ }^{108}$ J. Flix,${ }^{108}$ M. C. Fouz,${ }^{108}$ P. Garcia-Abia, ${ }^{108}$ O. Gonzalez Lopez, ${ }^{108}$ S. Goy Lopez, ${ }^{108}$ J. M. Hernandez, ${ }^{108}$ M. I. Josa, ${ }^{108}$ A. Pérez-Calero Yzquierdo, ${ }^{108}$ J. Puerta Pelayo, ${ }^{108}$ A. Quintario Olmeda, ${ }^{108}$ I. Redondo, ${ }^{108}$ L. Romero, ${ }^{108}$ M. S. Soares, ${ }^{108}$ A. Álvarez Fernández, ${ }^{108}$ J. F. de Trocóniz, ${ }^{109}$ M. Missiroli, ${ }^{109}$ D. Moran, ${ }^{109}$ J. Cuevas, ${ }^{110}$ C. Erice, ${ }^{110}$ J. Fernandez Menendez,${ }^{110}$ I. Gonzalez Caballero, ${ }^{110}$ J. R. González Fernández, ${ }^{110}$ E. Palencia Cortezon, ${ }^{110}$ S. Sanchez Cruz, ${ }^{110}$ I. Suárez Andrés, ${ }^{110}$ P. Vischia, ${ }^{110}$ J. M. Vizan Garcia,${ }^{110}$ I. J. Cabrillo, ${ }^{111}$ A. Calderon, ${ }^{111}$ B. Chazin Quero, ${ }^{111}$ E. Curras, ${ }^{111}$ M. Fernandez, ${ }^{111}$ J. Garcia-Ferrero, ${ }^{111}$ G. Gomez, ${ }^{111}$ A. Lopez Virto, ${ }^{111}$ J. Marco, ${ }^{111}$ C. Martinez Rivero, ${ }^{111}$ P. Martinez Ruiz del Arbol, ${ }^{111}$ F. Matorras, ${ }^{111}$ J. Piedra Gomez, ${ }^{111}$ T. Rodrigo, ${ }^{111}$ A. Ruiz-Jimeno, ${ }^{111}$ L. Scodellaro, ${ }^{111}$ N. Trevisani, ${ }^{111}$ I. Vila, ${ }^{111}$ R. Vilar Cortabitarte, ${ }^{111}$ D. Abbaneo, ${ }^{112}$ E. Auffray, ${ }^{112}$ P. Baillon, ${ }^{112}$ A. H. Ball, ${ }^{112}$ D. Barney, ${ }^{112}$ M. Bianco, ${ }^{112}$ P. Bloch,${ }^{112}$ A. Bocci,${ }^{112}$ C. Botta, ${ }^{112}$ T. Camporesi, ${ }^{112}$ R. Castello, ${ }^{112}$ M. Cepeda, ${ }^{112}$ G. Cerminara, ${ }^{112}$ E. Chapon, ${ }^{112}$ Y. Chen, ${ }^{112}$ D. d'Enterria, ${ }^{112}$ A. Dabrowski, ${ }^{112}$ V. Daponte, ${ }^{112}$ A. David, ${ }^{112}$ M. De Gruttola, ${ }^{112}$ A. De Roeck, ${ }^{112}$ E. Di Marco, ${ }^{12, \text { ss }}$ M. Dobson, ${ }^{112}$ B. Dorney, ${ }^{112}$ T. du Pree, ${ }^{112}$ M. Dünser, ${ }^{112}$ N. Dupont ${ }^{112}$ A. Elliott-Peisert, ${ }^{112}$ P. Everaerts, ${ }^{112}$ G. Franzoni, ${ }^{112}$ J. Fulcher, ${ }^{112}$ W. Funk,${ }^{112}$ D. Gigi,${ }^{112}$ K. Gill, ${ }^{112}$ F. Glege, ${ }^{112}$ D. Gulhan, ${ }^{112}$ S. Gundacker, ${ }^{112}$ M. Guthoff, ${ }^{112}$ P. Harris, ${ }^{112}$ J. Hegeman, ${ }^{112}$ V. Innocente, ${ }^{112}$ P. Janot,,${ }^{112}$ O. Karacheban, ${ }^{112, \mathrm{~s}}$ J. Kieseler, ${ }^{112}$ H. Kirschenmann, ${ }^{112}$ V. Knünz, ${ }^{112}$ A. Kornmayer, ${ }^{112, \mathrm{p}}$ M. J. Kortelainen, ${ }^{112}$ C. Lange, ${ }^{112}$ P. Lecoq ${ }^{112}$ C. Lourenço, ${ }^{112}$ M. T. Lucchini, ${ }^{112}$ L. Malgeri, ${ }^{112}$ M. Mannelli, ${ }^{112}$ A. Martelli, ${ }^{112}$ F. Meijers, ${ }^{112}$ J. A. Merlin, ${ }^{112}$ S. Mersi, ${ }^{112}$ E. Meschi, ${ }^{112}$ P. Milenovic, ${ }^{112, t t}$ F. Moortgat, ${ }^{112}$ M. Mulders, ${ }^{112}$ H. Neugebauer, ${ }^{112}$ S. Orfanelli, ${ }^{112}$ L. Orsini, ${ }^{112}$ L. Pape,${ }^{112}$ E. Perez, ${ }^{112}$ M. Peruzzi, ${ }^{112}$ A. Petrilli, ${ }^{112}$ G. Petrucciani, ${ }^{112}$ A. Pfeiffer, ${ }^{112}$ M. Pierini, ${ }^{112}$ A. Racz, ${ }^{112}$ T. Reis, ${ }^{112}$ G. Rolandi, ${ }^{112, \text { uu }}$ M. Rovere, ${ }^{112}$ H. Sakulin, ${ }^{112}$ C. Schäfer, ${ }^{112}$ C. Schwick,${ }^{112}$ M. Seidel, ${ }^{112}$ M. Selvaggi, ${ }^{112}$ A. Sharma, ${ }^{112}$

P. Silva, ${ }^{112}$ P. Sphicas, ${ }^{112, v v}$ J. Steggemann, ${ }^{112}$ M. Stoye, ${ }^{112}$ M. Tosi, ${ }^{112}$ D. Treille,${ }^{112}$ A. Triossi, ${ }^{112}$ A. Tsirou, ${ }^{112}$ V. Veckalns, ${ }^{112, w w}$ G. I. Veres, ${ }^{112, \mathrm{u}}$ M. Verweij, ${ }^{112}$ N. Wardle, ${ }^{112}$ W. D. Zeuner, ${ }^{112}$ W. Bertl, ${ }^{113, a}$ K. Deiters, ${ }^{113}$ W. Erdmann, ${ }^{113}$ R. Horisberger, ${ }^{113}$ Q. Ingram, ${ }^{113}$ H. C. Kaestli, ${ }^{113}$ D. Kotlinski, ${ }^{113}$ U. Langenegger, ${ }^{113}$ T. Rohe, ${ }^{113}$ S. A. Wiederkehr, ${ }^{113}$ F. Bachmair, ${ }^{114}$ L. Bäni,${ }^{114}$ P. Berger,${ }^{114}$ L. Bianchini, ${ }^{114}$ B. Casal, ${ }^{114}$ G. Dissertori, ${ }^{114}$ M. Dittmar, ${ }^{114}$ M. Donegà,${ }^{114}$ C. Grab, ${ }^{114}$ C. Heidegger, ${ }^{114}$ D. Hits, ${ }^{114}$ J. Hoss, ${ }^{114}$ G. Kasieczka, ${ }^{114}$ T. Klijnsma, ${ }^{114}$ W. Lustermann, ${ }^{114}$ B. Mangano, ${ }^{114}$ M. Marionneau, ${ }^{114}$ M. T. Meinhard, ${ }^{114}$ D. Meister, ${ }^{114}$ F. Micheli, ${ }^{114}$ P. Musella ${ }^{114}$ F. Nessi-Tedaldi, ${ }^{114}$ F. Pandolfi, ${ }^{114}$ 
J. Pata, ${ }^{114}$ F. Pauss, ${ }^{114}$ G. Perrin, ${ }^{114}$ L. Perrozzi, ${ }^{114}$ M. Quittnat, ${ }^{114}$ M. Schönenberger, ${ }^{114}$ L. Shchutska, ${ }^{114}$ A. Starodumov, ${ }^{114, \mathrm{xx}}$ V. R. Tavolaro, ${ }^{114}$ K. Theofilatos, ${ }^{114}$ M. L. Vesterbacka Olsson, ${ }^{114}$ R. Wallny, ${ }^{114}$ A. Zagozdzinska, ${ }^{114, j j}$ D. H. Zhu, ${ }^{114}$ T. K. Aarrestad, ${ }^{115}$ C. Amsler, ${ }^{15, y y}$ L. Caminada, ${ }^{115}$ M. F. Canelli, ${ }^{115}$ A. De Cosa, ${ }^{15}$ S. Donato, ${ }^{115}$ C. Galloni, ${ }^{115}$ T. Hreus, ${ }^{115}$ B. Kilminster, ${ }^{115}$ J. Ngadiuba, ${ }^{115}$ D. Pinna, ${ }^{115}$ G. Rauco, ${ }^{115}$ P. Robmann, ${ }^{115}$ D. Salerno, ${ }^{115}$ C. Seitz, ${ }^{115}$ A. Zucchetta, ${ }^{115}$ V. Candelise, ${ }^{116}$ T. H. Doan, ${ }^{116}$ Sh. Jain, ${ }^{116}$ R. Khurana, ${ }^{116}$ C. M. Kuo, ${ }^{116}$ W. Lin, ${ }^{116}$ A. Pozdnyakov, ${ }^{116}$ S. S. Yu, ${ }^{116}$ Arun Kumar, ${ }^{117}$ P. Chang, ${ }^{117}$ Y. Chao,${ }^{117}$ K. F. Chen, ${ }^{117}$ P. H. Chen,${ }^{117}$ F. Fiori, ${ }^{117}$ W.-S. Hou, ${ }^{117}$ Y. Hsiung, ${ }^{117}$ Y. F. Liu, ${ }^{117}$ R.-S. Lu, ${ }^{117}$ M. Miñano Moya,${ }^{117}$ E. Paganis, ${ }^{117}$ A. Psallidas, ${ }^{117}$ J. f. Tsai,${ }^{117}$ B. Asavapibhop, ${ }^{118}$ K. Kovitanggoon, ${ }^{118}$ G. Singh, ${ }^{118}$ N. Srimanobhas, ${ }^{118}$ A. Adiguzel, ${ }^{119, z z}$ F. Boran, ${ }^{119}$ S. Cerci, ${ }^{19, \text { aaa }}$ S. Damarseckin, ${ }^{119}$ Z. S. Demiroglu, ${ }^{119}$ C. Dozen, ${ }^{119}$ I. Dumanoglu, ${ }^{119}$ S. Girgis, ${ }^{119}$ G. Gokbulut, ${ }^{119}$ Y. Guler, ${ }^{119}$ I. Hos, ${ }^{19, \text { bbb }}$ E. E. Kangal, ${ }^{119, \text { ccc }}$ O. Kara, ${ }^{119}$ U. Kiminsu, ${ }^{119}$ M. Oglakci, ${ }^{19}$ G. Onengut, ${ }^{11, \text { ddd }}$ K. Ozdemir, ${ }^{19, \text { eee }}$ D. Sunar Cerci, ${ }^{119 \text {,aaa }}$ B. Tali, ${ }^{119, \text { aaa }}$ H. Topakli, ${ }^{11, \text { fff }}$ S. Turkcapar, ${ }^{119}$ I. S. Zorbakir, ${ }^{119}$ C. Zorbilmez, ${ }^{119}$ B. Bilin, ${ }^{120}$ G. Karapinar, ${ }^{120, g g g}$ K. Ocalan, ${ }^{120, \text { hhh }}$ M. Yalvac, ${ }^{120}$ M. Zeyrek, ${ }^{120}$ E. Gülmez, ${ }^{121}$ M. Kaya, ${ }^{121, \text { iii }}$ O. Kaya,${ }^{121, j j j}$ S. Tekten, ${ }^{121}$ E. A. Yetkin, ${ }^{121, k k k}$ M. N. Agaras, ${ }^{122}$ S. Atay, ${ }^{122}$ A. Cakir, ${ }^{122}$ K. Cankocak, ${ }^{122}$ B. Grynyov, ${ }^{123}$ L. Levchuk, ${ }^{124}$ P. Sorokin, ${ }^{124}$ R. Aggleton, ${ }^{125}$ F. Ball, ${ }^{125}$ L. Beck, ${ }^{125}$ J. J. Brooke, ${ }^{125}$ D. Burns, ${ }^{125}$ E. Clement, ${ }^{125}$ D. Cussans, ${ }^{125}$ O. Davignon, ${ }^{125}$ H. Flacher, ${ }^{125}$ J. Goldstein, ${ }^{125}$ M. Grimes, ${ }^{125}$ G. P. Heath, ${ }^{125}$ H. F. Heath, ${ }^{125}$ J. Jacob, ${ }^{125}$ L. Kreczko, ${ }^{125}$ C. Lucas, ${ }^{125}$ D. M. Newbold, ${ }^{125,111}$ S. Paramesvaran, ${ }^{125}$ A. Poll, ${ }^{125}$ T. Sakuma, ${ }^{125}$ S. Seif El Nasr-storey, ${ }^{125}$ D. Smith, ${ }^{125}$ V. J. Smith, ${ }^{125}$ K. W. Bell, ${ }^{126}$ A. Belyaev, ${ }^{126, m m m}$ C. Brew, ${ }^{126}$ R. M. Brown, ${ }^{126}$ L. Calligaris, ${ }^{126}$ D. Cieri, ${ }^{126}$ D. J. A. Cockerill, ${ }^{126}$ J. A. Coughlan, ${ }^{126}$ K. Harder, ${ }^{126}$ S. Harper, ${ }^{126}$ E. Olaiya, ${ }^{126}$ D. Petyt, ${ }^{126}$ C. H. Shepherd-Themistocleous, ${ }^{126}$ A. Thea, ${ }^{126}$ I. R. Tomalin, ${ }^{126}$ T. Williams, ${ }^{126}$ R. Bainbridge, ${ }^{127}$ S. Breeze, ${ }^{127}$ O. Buchmuller, ${ }^{127}$ A. Bundock, ${ }^{127}$ S. Casasso, ${ }^{127}$ M. Citron, ${ }^{127}$ D. Colling, ${ }^{127}$ L. Corpe,${ }^{127}$ P. Dauncey, ${ }^{127}$ G. Davies, ${ }^{127}$ A. De Wit, ${ }^{127}$ M. Della Negra, ${ }^{127}$ R. Di Maria, ${ }^{127}$ A. Elwood, ${ }^{127}$ D. Futyan, ${ }^{127}$ Y. Haddad, ${ }^{127}$ G. Hall, ${ }^{127}$ G. Iles, ${ }^{127}$ T. James, ${ }^{127}$ R. Lane, ${ }^{127}$ C. Laner, ${ }^{127}$ L. Lyons, ${ }^{127}$ A.-M. Magnan, ${ }^{127}$ S. Malik, ${ }^{127}$ L. Mastrolorenzo, ${ }^{127}$ T. Matsushita, ${ }^{127}$ J. Nash, ${ }^{127}$ A. Nikitenko, ${ }^{127, x x}$ V. Palladino, ${ }^{127}$ M. Pesaresi, ${ }^{127}$ D. M. Raymond, ${ }^{127}$ A. Richards, ${ }^{127}$ A. Rose, ${ }^{127}$ E. Scott, ${ }^{127}$ C. Seez, ${ }^{127}$ A. Shtipliyski, ${ }^{127}$ S. Summers, ${ }^{127}$ A. Tapper ${ }^{127}$ K. Uchida, ${ }^{127}$ M. Vazquez Acosta, ${ }^{127, n n n}$ T. Virdee, ${ }^{127, p}$ D. Winterbottom, ${ }^{127}$ J. Wright, ${ }^{127}$ S. C. Zenz, ${ }^{127}$ J. E. Cole, ${ }^{128}$ P. R. Hobson, ${ }^{128}$ A. Khan, ${ }^{128}$ P. Kyberd, ${ }^{128}$ I. D. Reid, ${ }^{128}$ P. Symonds, ${ }^{128}$ L. Teodorescu, ${ }^{128}$ M. Turner, ${ }^{128}$ A. Borzou, ${ }^{129}$ K. Call, ${ }^{129}$ J. Dittmann, ${ }^{129}$ K. Hatakeyama, ${ }^{129}$ H. Liu, ${ }^{129}$ N. Pastika, ${ }^{129}$ R. Bartek, ${ }^{130}$ A. Dominguez, ${ }^{130}$ A. Buccilli, ${ }^{131}$ S. I. Cooper, ${ }^{131}$ C. Henderson, ${ }^{131}$ P. Rumerio, ${ }^{131}$ C. West, ${ }^{131}$ D. Arcaro, ${ }^{132}$ A. Avetisyan, ${ }^{132}$ T. Bose, ${ }^{132}$ D. Gastler, ${ }^{132}$ D. Rankin, ${ }^{132}$ C. Richardson, ${ }^{132}$ J. Rohlf, ${ }^{132}$ L. Sulak,${ }^{132}$ D. Zou, ${ }^{132}$ G. Benelli, ${ }^{133}$ D. Cutts, ${ }^{133}$

A. Garabedian, ${ }^{133}$ J. Hakala, ${ }^{133}$ U. Heintz, ${ }^{133}$ J. M. Hogan, ${ }^{133}$ K. H. M. Kwok, ${ }^{133}$ E. Laird, ${ }^{133}$ G. Landsberg, ${ }^{133}$ Z. Mao, ${ }^{133}$ M. Narain, ${ }^{133}$ S. Piperov, ${ }^{133}$ S. Sagir, ${ }^{133}$ R. Syarif, ${ }^{133}$ D. Yu, ${ }^{133}$ R. Band, ${ }^{134}$ C. Brainerd, ${ }^{134}$ D. Burns, ${ }^{134}$ M. Calderon De La Barca Sanchez, ${ }^{134}$ M. Chertok, ${ }^{134}$ J. Conway, ${ }^{134}$ R. Conway, ${ }^{134}$ P. T. Cox, ${ }^{134}$ R. Erbacher, ${ }^{134}$ C. Flores, ${ }^{134}$ G. Funk, ${ }^{134}$ M. Gardner, ${ }^{134}$ W. Ko, ${ }^{134}$ R. Lander, ${ }^{134}$ C. Mclean, ${ }^{134}$ M. Mulhearn, ${ }^{134}$ D. Pellett, ${ }^{134}$ J. Pilot, ${ }^{134}$ S. Shalhout, ${ }^{134}$ M. Shi, ${ }^{134}$ J. Smith, ${ }^{134}$ M. Squires, ${ }^{134}$ D. Stolp, ${ }^{134}$ K. Tos, ${ }^{134}$ M. Tripathi, ${ }^{134}$ Z. Wang, ${ }^{134}$ M. Bachtis, ${ }^{135}$ C. Bravo, ${ }^{135}$ R. Cousins,${ }^{135}$ A. Dasgupta, ${ }^{135}$ A. Florent, ${ }^{135}$ J. Hauser, ${ }^{135}$ M. Ignatenko, ${ }^{135}$ N. Mccoll, ${ }^{135}$ D. Saltzberg, ${ }^{135}$ C. Schnaible,${ }^{135}$ V. Valuev, ${ }^{135}$ E. Bouvier, ${ }^{136}$ K. Burt, ${ }^{136}$ R. Clare, ${ }^{136}$ J. Ellison, ${ }^{136}$ J. W. Gary, ${ }^{136}$ S. M. A. Ghiasi Shirazi,${ }^{136}$ G. Hanson, ${ }^{136}$ J. Heilman, ${ }^{136}$ P. Jandir, ${ }^{136}$ E. Kennedy, ${ }^{136}$ F. Lacroix, ${ }^{136}$ O. R. Long, ${ }^{136}$ M. Olmedo Negrete, ${ }^{136}$ M. I. Paneva, ${ }^{136}$ A. Shrinivas, ${ }^{136}$ W. Si ${ }^{136}$ L. Wang, ${ }^{136}$ H. Wei, ${ }^{136}$ S. Wimpenny, ${ }^{136}$ B. R. Yates, ${ }^{136}$ J. G. Branson, ${ }^{137}$ S. Cittolin, ${ }^{137}$ M. Derdzinski, ${ }^{137}$ B. Hashemi, ${ }^{137}$ A. Holzner, ${ }^{137}$ D. Klein, ${ }^{137}$ G. Kole, ${ }^{137}$ V. Krutelyov, ${ }^{137}$ J. Letts, ${ }^{137}$ I. Macneill, ${ }^{137}$ M. Masciovecchio, ${ }^{137}$ D. Olivito, ${ }^{137}$ S. Padhi,${ }^{137}$ M. Pieri, ${ }^{137}$ M. Sani, ${ }^{137}$ V. Sharma, ${ }^{137}$ S. Simon, ${ }^{137}$ M. Tadel,${ }^{137}$ A. Vartak, ${ }^{137}$ S. Wasserbaech, ${ }^{137,0 o o}$ J. Wood,${ }^{137}$ F. Würthwein, ${ }^{137}$ A. Yagil, ${ }^{137}$ G. Zevi Della Porta, ${ }^{137}$ N. Amin, ${ }^{138}$ R. Bhandari, ${ }^{138}$ J. Bradmiller-Feld, ${ }^{138}$ C. Campagnari, ${ }^{138}$ A. Dishaw, ${ }^{138}$ V. Dutta, ${ }^{138}$ M. Franco Sevilla, ${ }^{138}$ C. George,${ }^{138}$ F. Golf, ${ }^{138}$ L. Gouskos, ${ }^{138}$ J. Gran, ${ }^{138}$ R. Heller, ${ }^{138}$ J. Incandela, ${ }^{138}$ S. D. Mullin, ${ }^{138}$ A. Ovcharova, ${ }^{138}$ A. Patterson, ${ }^{138}$ H. Qu, ${ }^{138}$ J. Richman, ${ }^{138}$ D. Stuart, ${ }^{138}$ I. Suarez, ${ }^{138}$ J. Yoo, ${ }^{138}$ D. Anderson, ${ }^{139}$ J. Bendavid, ${ }^{139}$ A. Bornheim, ${ }^{139}$ J. M. Lawhorn, ${ }^{139}$ H. B. Newman, ${ }^{139}$ T. Nguyen, ${ }^{139}$ C. Pena, ${ }^{139}$ M. Spiropulu, ${ }^{139}$ J. R. Vlimant, ${ }^{139}$ S. Xie, ${ }^{139}$ Z. Zhang, ${ }^{139}$ R. Y. Zhu, ${ }^{139}$ M. B. Andrews, ${ }^{140}$ T. Ferguson, ${ }^{140}$ T. Mudholkar, ${ }^{140}$ M. Paulini, ${ }^{140}$ J. Russ, ${ }^{140}$ M. Sun, ${ }^{140}$ H. Vogel, ${ }^{140}$ I. Vorobiev, ${ }^{140}$ M. Weinberg, ${ }^{140}$ J. P. Cumalat, ${ }^{141}$ W. T. Ford, ${ }^{141}$ F. Jensen, ${ }^{141}$ A. Johnson, ${ }^{141}$ M. Krohn, ${ }^{141}$ S. Leontsinis, ${ }^{141}$ T. Mulholland, ${ }^{141}$ K. Stenson, ${ }^{141}$ S. R. Wagner, ${ }^{141}$ J. Alexander, ${ }^{142}$ J. Chaves, ${ }^{142}$ J. Chu, ${ }^{142}$ S. Dittmer, ${ }^{142}$ K. Mcdermott, ${ }^{142}$ N. Mirman, ${ }^{142}$ J. R. Patterson, ${ }^{142}$ A. Rinkevicius, ${ }^{142}$ A. Ryd ${ }^{142}$ L. Skinnari,${ }^{142}$ L. Soffi, ${ }^{142}$ S. M. Tan, ${ }^{142}$ Z. Tao, ${ }^{142}$ J. Thom, ${ }^{142}$ J. Tucker, ${ }^{142}$ P. Wittich, ${ }^{142}$ M. Zientek, ${ }^{142}$ S. Abdullin, ${ }^{143}$ M. Albrow, ${ }^{143}$ G. Apollinari, ${ }^{143}$ A. Apresyan, ${ }^{143}$ 
A. Apyan, ${ }^{143}$ S. Banerjee, ${ }^{143}$ L. A. T. Bauerdick, ${ }^{143}$ A. Beretvas, ${ }^{143}$ J. Berryhill, ${ }^{143}$ P. C. Bhat,${ }^{143}$ G. Bolla, ${ }^{143}$ K. Burkett, ${ }^{143}$ J. N. Butler, ${ }^{143}$ A. Canepa ${ }^{143}$ G. B. Cerati, ${ }^{143}$ H. W. K. Cheung, ${ }^{143}$ F. Chlebana, ${ }^{143}$ M. Cremonesi, ${ }^{143}$ J. Duarte, ${ }^{143}$ V. D. Elvira, ${ }^{143}$ J. Freeman, ${ }^{143}$ Z. Gecse ${ }^{143}$ E. Gottschalk, ${ }^{143}$ L. Gray, ${ }^{143}$ D. Green, ${ }^{143}$ S. Grünendahl, ${ }^{143}$ O. Gutsche, ${ }^{143}$ R. M. Harris, ${ }^{143}$ S. Hasegawa, ${ }^{143}$ J. Hirschauer, ${ }^{143}$ Z. Hu, ${ }^{143}$ B. Jayatilaka, ${ }^{143}$ S. Jindariani, ${ }^{143}$ M. Johnson, ${ }^{143}$ U. Joshi, ${ }^{143}$ B. Klima, ${ }^{143}$ B. Kreis, ${ }^{143}$ S. Lammel, ${ }^{143}$ D. Lincoln, ${ }^{143}$ R. Lipton, ${ }^{143}$ M. Liu, ${ }^{143}$ T. Liu, ${ }^{143}$ R. Lopes De Sá, ${ }^{143}$ J. Lykken, ${ }^{143}$

K. Maeshima, ${ }^{143}$ N. Magini, ${ }^{143}$ J. M. Marraffino, ${ }^{143}$ S. Maruyama, ${ }^{143}$ D. Mason, ${ }^{143}$ P. McBride,${ }^{143}$ P. Merkel, ${ }^{143}$ S. Mrenna, ${ }^{143}$ S. Nahn, ${ }^{143}$ V. O’Dell, ${ }^{143}$ K. Pedro, ${ }^{143}$ O. Prokofyev, ${ }^{143}$ G. Rakness, ${ }^{143}$ L. Ristori, ${ }^{143}$ B. Schneider, ${ }^{143}$ E. Sexton-Kennedy, ${ }^{143}$ A. Soha, ${ }^{143}$ W. J. Spalding, ${ }^{143}$ L. Spiegel, ${ }^{143}$ S. Stoynev ${ }^{143}$ J. Strait, ${ }^{143}$ N. Strobbe,${ }^{143}$ L. Taylor, ${ }^{143}$ S. Tkaczyk, ${ }^{143}$ N. V. Tran, ${ }^{143}$ L. Uplegger, ${ }^{143}$ E. W. Vaandering, ${ }^{143}$ C. Vernieri, ${ }^{143}$ M. Verzocchi, ${ }^{143}$ R. Vidal, ${ }^{143}$ M. Wang, ${ }^{143}$ H. A. Weber, ${ }^{143}$ A. Whitbeck, ${ }^{143}$ D. Acosta, ${ }^{144}$ P. Avery, ${ }^{144}$ P. Bortignon, ${ }^{144}$ D. Bourilkov, ${ }^{144}$ A. Brinkerhoff,,${ }^{144}$ A. Carnes, ${ }^{144}$ M. Carver, ${ }^{144}$ D. Curry, ${ }^{144}$ S. Das, ${ }^{144}$ R. D. Field, ${ }^{144}$ I. K. Furic, ${ }^{144}$ J. Konigsberg, ${ }^{144}$ A. Korytov, ${ }^{144}$ K. Kotov, ${ }^{144}$ P. Ma, ${ }^{144}$ K. Matchev, ${ }^{144}$ H. Mei, ${ }^{144}$ G. Mitselmakher, ${ }^{144}$ D. Rank, ${ }^{144}$ D. Sperka, ${ }^{144}$ N. Terentyev, ${ }^{144}$ L. Thomas,${ }^{144}$ J. Wang, ${ }^{144}$ S. Wang, ${ }^{144}$ J. Yelton, ${ }^{144}$ Y. R. Joshi, ${ }^{145}$ S. Linn, ${ }^{145}$ P. Markowitz, ${ }^{145}$ G. Martinez, ${ }^{145}$ J. L. Rodriguez, ${ }^{145}$ A. Ackert, ${ }^{146}$ T. Adams, ${ }^{146}$ A. Askew, ${ }^{146}$ S. Hagopian, ${ }^{146}$ V. Hagopian, ${ }^{146}$ K. F. Johnson, ${ }^{146}$ T. Kolberg, ${ }^{146}$ T. Perry, ${ }^{146}$ H. Prosper, ${ }^{146}$ A. Santra ${ }^{146}$ R. Yohay, ${ }^{146}$ M. M. Baarmand, ${ }^{147}$ V. Bhopatkar, ${ }^{147}$ S. Colafranceschi, ${ }^{147}$ M. Hohlmann, ${ }^{147}$ D. Noonan, ${ }^{147}$ T. Roy, ${ }^{147}$ F. Yumiceva, ${ }^{147}$ M. R. Adams, ${ }^{148}$ L. Apanasevich, ${ }^{148}$ D. Berry,${ }^{148}$ R. R. Betts, ${ }^{148}$ R. Cavanaugh, ${ }^{148}$ X. Chen, ${ }^{148}$ O. Evdokimov, ${ }^{148}$ C. E. Gerber, ${ }^{148}$ D. A. Hangal, ${ }^{148}$ D. J. Hofman, ${ }^{148}$ K. Jung, ${ }^{148}$ J. Kamin, ${ }^{148}$ I. D. Sandoval Gonzalez, ${ }^{148}$ M. B. Tonjes, ${ }^{148}$ H. Trauger, ${ }^{148}$ N. Varelas, ${ }^{148}$ H. Wang, ${ }^{148}$ Z. Wu, ${ }^{148}$ J. Zhang, ${ }^{148}$ B. Bilki, ${ }^{149, p p p}$ W. Clarida, ${ }^{149}$ K. Dilsiz, ${ }^{149, q q 9}$ S. Durgut, ${ }^{149}$ R. P. Gandrajula, ${ }^{149}$ M. Haytmyradov, ${ }^{149}$ V. Khristenko, ${ }^{149}$ J.-P. Merlo, ${ }^{149}$ H. Mermerkaya, ${ }^{149, \text { rrr }}$ A. Mestvirishvili, ${ }^{149}$ A. Moeller, ${ }^{149}$ J. Nachtman, ${ }^{149}$ H. Ogul, ${ }^{149, \text { sss }}$ Y. Onel, ${ }^{149}$ F. Ozok, ${ }^{149, \text { ttt }}$ A. Penzo, ${ }^{149}$ C. Snyder, ${ }^{149}$ E. Tiras, ${ }^{149}$ J. Wetzel,${ }^{149}$ K. Yi, ${ }^{149}$ B. Blumenfeld, ${ }^{150}$ A. Cocoros, ${ }^{150}$ N. Eminizer, ${ }^{150}$ D. Fehling, ${ }^{150}$ L. Feng, ${ }^{150}$ A. V. Gritsan, ${ }^{150}$ P. Maksimovic, ${ }^{150}$ C. Mantilla, ${ }^{150}$ J. Roskes, ${ }^{150}$ U. Sarica, ${ }^{150}$ M. Swartz, ${ }^{150}$ M. Xiao, ${ }^{150}$ C. You, ${ }^{150}$ A. Al-bataineh, ${ }^{151}$ P. Baringer, ${ }^{151}$ A. Bean, ${ }^{151}$ S. Boren,${ }^{151}$ J. Bowen, ${ }^{151}$ J. Castle, ${ }^{151}$ S. Khalil,${ }^{151}$ A. Kropivnitskaya, ${ }^{151}$ D. Majumder, ${ }^{151}$ W. Mcbrayer,${ }^{151}$ M. Murray ${ }^{151}$ C. Royon, ${ }^{151}$ S. Sanders, ${ }^{151}$ E. Schmitz,${ }^{151}$ R. Stringer ${ }^{151}$ J. D. Tapia Takaki, ${ }^{151}$ Q. Wang, ${ }^{151}$ A. Ivanov, ${ }^{152}$ K. Kaadze, ${ }^{152}$ Y. Maravin, ${ }^{152}$ A. Mohammadi, ${ }^{152}$ L. K. Saini ${ }^{152}$ N. Skhirtladze, ${ }^{152}$ S. Toda, ${ }^{152}$ F. Rebassoo, ${ }^{153}$ D. Wright, ${ }^{153}$ C. Anelli, ${ }^{154}$ A. Baden, ${ }^{154}$ O. Baron, ${ }^{154}$ A. Belloni, ${ }^{154}$ B. Calvert, ${ }^{154}$ S. C. Eno, ${ }^{154}$ C. Ferraioli, ${ }^{154}$ N. J. Hadley, ${ }^{154}$ S. Jabeen, ${ }^{154}$ G. Y. Jeng, ${ }^{154}$ R. G. Kellogg, ${ }^{154}$ J. Kunkle, ${ }^{154}$ A. C. Mignerey, ${ }^{154}$ F. Ricci-Tam, ${ }^{154}$ Y. H. Shin, ${ }^{154}$ A. Skuja ${ }^{154}$ S. C. Tonwar, ${ }^{154}$ D. Abercrombie, ${ }^{155}$ B. Allen, ${ }^{155}$ V. Azzolini, ${ }^{155}$ R. Barbieri, ${ }^{155}$ A. Baty, ${ }^{155}$ R. Bi ${ }^{155}$ S. Brandt, ${ }^{155}$ W. Busza, ${ }^{155}$ I. A. Cali, ${ }^{155}$ M. D' Alfonso, ${ }^{155}$ Z. Demiragli, ${ }^{155}$ G. Gomez Ceballos, ${ }^{155}$ M. Goncharov, ${ }^{155}$ D. Hsu, ${ }^{155}$ Y. Iiyama, ${ }^{155}$ G. M. Innocenti, ${ }^{155}$ M. Klute, ${ }^{155}$ D. Kovalskyi, ${ }^{155}$ Y. S. Lai, ${ }^{155}$ Y.-J. Lee, ${ }^{155}$ A. Levin, ${ }^{155}$ P. D. Luckey, ${ }^{155}$ B. Maier, ${ }^{155}$ A. C. Marini, ${ }^{155}$ C. Mcginn,${ }^{155}$ C. Mironov, ${ }^{155}$ S. Narayanan, ${ }^{155}$ X. Niu, ${ }^{155}$ C. Paus, ${ }^{155}$ C. Roland,${ }^{155}$ G. Roland, ${ }^{155}$ J. Salfeld-Nebgen,,${ }^{155}$

G. S. F. Stephans, ${ }^{155}$ K. Tatar, ${ }^{155}$ D. Velicanu, ${ }^{155}$ J. Wang, ${ }^{155}$ T. W. Wang ${ }^{155}$ B. Wyslouch, ${ }^{155}$ A. C. Benvenuti, ${ }^{156}$ R. M. Chatterjee, ${ }^{156}$ A. Evans, ${ }^{156}$ P. Hansen, ${ }^{156}$ S. Kalafut, ${ }^{156}$ Y. Kubota, ${ }^{156}$ Z. Lesko, ${ }^{156}$ J. Mans, ${ }^{156}$ S. Nourbakhsh, ${ }^{156}$ N. Ruckstuhl, ${ }^{156}$ R. Rusack, ${ }^{156}$ J. Turkewitz, ${ }^{156}$ J. G. Acosta, ${ }^{157}$ S. Oliveros, ${ }^{157}$ E. Avdeeva, ${ }^{158}$ K. Bloom, ${ }^{158}$ D. R. Claes, ${ }^{158}$ C. Fangmeier, ${ }^{158}$ R. Gonzalez Suarez, ${ }^{158}$ R. Kamalieddin, ${ }^{158}$ I. Kravchenko, ${ }^{158}$ J. Monroy, ${ }^{158}$ J. E. Siado, ${ }^{158}$ G. R. Snow, ${ }^{158}$ B. Stieger, ${ }^{158}$ M. Alyari, ${ }^{159}$ J. Dolen, ${ }^{159}$ A. Godshalk, ${ }^{159}$ C. Harrington, ${ }^{159}$ I. Iashvili ${ }^{159}$ D. Nguyen, ${ }^{159}$ A. Parker,${ }^{159}$ S. Rappoccio ${ }^{159}$ B. Roozbahani, ${ }^{159}$ G. Alverson, ${ }^{160}$ E. Barberis, ${ }^{160}$ A. Hortiangtham, ${ }^{160}$ A. Massironi, ${ }^{160}$ D. M. Morse, ${ }^{160}$ D. Nash, ${ }^{160}$ T. Orimoto, ${ }^{160}$ R. Teixeira De Lima, ${ }^{160}$ D. Trocino, ${ }^{160}$ R.-J. Wang, ${ }^{160}$ D. Wood, ${ }^{160}$ S. Bhattacharya, ${ }^{161}$ O. Charaf, ${ }^{161}$ K. A. Hahn, ${ }^{161}$ N. Mucia, ${ }^{161}$ N. Odell, ${ }^{161}$ B. Pollack, ${ }^{161}$ M. H. Schmitt, ${ }^{161}$ K. Sung, ${ }^{161}$ M. Trovato, ${ }^{161}$ M. Velasco, ${ }^{161}$ N. Dev, ${ }^{162}$ M. Hildreth, ${ }^{162}$ K. Hurtado Anampa, ${ }^{162}$ C. Jessop, ${ }^{162}$ D. J. Karmgard, ${ }^{162}$ N. Kellams, ${ }^{162}$ K. Lannon, ${ }^{162}$ N. Loukas, ${ }^{162}$ N. Marinelli, ${ }^{162}$ F. Meng, ${ }^{162}$ C. Mueller, ${ }^{162}$ Y. Musienko, ${ }^{162, k k}$ M. Planer, ${ }^{162}$ A. Reinsvold, ${ }^{162}$ R. Ruchti, ${ }^{162}$ G. Smith, ${ }^{162}$ S. Taroni,${ }^{162}$ M. Wayne, ${ }^{162}$ M. Wolf, ${ }^{162}$ A. Woodard,${ }^{162}$ J. Alimena, ${ }^{163}$ L. Antonelli, ${ }^{163}$ B. Bylsma ${ }^{163}$ L. S. Durkin, ${ }^{163}$ S. Flowers, ${ }^{163}$ B. Francis, ${ }^{163}$ A. Hart,${ }^{163}$ C. Hill, ${ }^{163}$ W. Ji, ${ }^{163}$ B. Liu, ${ }^{163}$ W. Luo, ${ }^{163}$ D. Puigh, ${ }^{163}$ B. L. Winer, ${ }^{163}$ H. W. Wulsin, ${ }^{163}$ A. Benaglia, ${ }^{164}$ S. Cooperstein, ${ }^{164}$ O. Driga, ${ }^{164}$ P. Elmer, ${ }^{164}$ J. Hardenbrook, ${ }^{164}$ P. Hebda, ${ }^{164}$ S. Higginbotham, ${ }^{164}$ D. Lange, ${ }^{164}$ J. Luo, ${ }^{164}$ D. Marlow, ${ }^{164}$ K. Mei, ${ }^{164}$ I. Ojalvo, ${ }^{164}$ J. Olsen, ${ }^{164}$ C. Palmer, ${ }^{164}$ P. Piroué, ${ }^{164}$ D. Stickland, ${ }^{164}$ C. Tully, ${ }^{164}$ S. Malik, ${ }^{165}$ S. Norberg, ${ }^{165}$ A. Barker, ${ }^{166}$ V. E. Barnes, ${ }^{166}$ S. Folgueras, ${ }^{166}$ L. Gutay, ${ }^{166}$ M. K. Jha, ${ }^{166}$ M. Jones, ${ }^{166}$ A. W. Jung, ${ }^{166}$ A. Khatiwada, ${ }^{166}$ D. H. Miller, ${ }^{166}$ N. Neumeister,${ }^{166}$ C. C. Peng,${ }^{166}$ J. F. Schulte, ${ }^{166}$ J. Sun, ${ }^{166}$ F. Wang, ${ }^{166}$ W. Xie, ${ }^{166}$ T. Cheng, ${ }^{167}$ N. Parashar, ${ }^{167}$ J. Stupak, ${ }^{167}$ A. Adair, ${ }^{168}$ B. Akgun, ${ }^{168}$ Z. Chen, ${ }^{168}$ 
K. M. Ecklund, ${ }^{168}$ F. J. M. Geurts, ${ }^{168}$ M. Guilbaud, ${ }^{168}$ W. Li, ${ }^{168}$ B. Michlin, ${ }^{168}$ M. Northup, ${ }^{168}$ B. P. Padley, ${ }^{168}$ J. Roberts, ${ }^{168}$ J. Rorie, ${ }^{168}$ Z. Tu, ${ }^{168}$ J. Zabel, ${ }^{168}$ A. Bodek, ${ }^{169}$ P. de Barbaro, ${ }^{169}$ R. Demina, ${ }^{169}$ Y. t. Duh, ${ }^{169}$ T. Ferbel, ${ }^{169}$ M. Galanti, ${ }^{169}$ A. Garcia-Bellido, ${ }^{169}$ J. Han, ${ }^{169}$ O. Hindrichs, ${ }^{169}$ A. Khukhunaishvili, ${ }^{169}$ K. H. Lo, ${ }^{169}$ P. Tan, ${ }^{169}$ M. Verzetti, ${ }^{169}$ R. Ciesielski, ${ }^{170}$ K. Goulianos, ${ }^{170}$ C. Mesropian, ${ }^{170}$ A. Agapitos, ${ }^{171}$ J. P. Chou, ${ }^{171}$ Y. Gershtein, ${ }^{171}$ T. A. Gómez Espinosa, ${ }^{171}$ E. Halkiadakis, ${ }^{171}$ M. Heindl, ${ }^{171}$ E. Hughes, ${ }^{171}$ S. Kaplan, ${ }^{171}$ R. Kunnawalkam Elayavalli, ${ }^{171}$ S. Kyriacou, ${ }^{171}$ A. Lath, ${ }^{171}$ R. Montalvo, ${ }^{171}$ K. Nash, ${ }^{171}$ M. Osherson,${ }^{171}$ H. Saka, ${ }^{171}$ S. Salur, ${ }^{171}$ S. Schnetzer, ${ }^{171}$ D. Sheffield, ${ }^{171}$ S. Somalwar, ${ }^{171}$ R. Stone,${ }^{171}$ S. Thomas, ${ }^{171}$ P. Thomassen, ${ }^{171}$ M. Walker, ${ }^{171}$ A. G. Delannoy, ${ }^{172}$ M. Foerster, ${ }^{172}$ J. Heideman, ${ }^{172}$ G. Riley, ${ }^{172}$ K. Rose ${ }^{172}$ S. Spanier, ${ }^{172}$ K. Thapa, ${ }^{172}$ O. Bouhali, ${ }^{173, \text { uuu }}$ A. Castaneda Hernandez, ${ }^{173, \text { uuu }}$ A. Celik, ${ }^{173}$ M. Dalchenko, ${ }^{173}$ M. De Mattia, ${ }^{173}$ A. Delgado, ${ }^{173}$ S. Dildick, ${ }^{173}$ R. Eusebi, ${ }^{173}$ J. Gilmore, ${ }^{173}$ T. Huang, ${ }^{173}$ T. Kamon, ${ }^{173, v v v}$ R. Mueller, ${ }^{173}$ Y. Pakhotin, ${ }^{173}$ R. Patel, ${ }^{173}$ A. Perloff, ${ }^{173}$ L. Perniè, ${ }^{173}$ D. Rathjens, ${ }^{173}$ A. Safonov, ${ }^{173}$ A. Tatarinov, ${ }^{173}$ K. A. Ulmer, ${ }^{173}$ N. Akchurin, ${ }^{174}$ J. Damgov, ${ }^{174}$ F. De Guio, ${ }^{174}$ P. R. Dudero, ${ }^{174}$ J. Faulkner, ${ }^{174}$ E. Gurpinar, ${ }^{174}$ S. Kunori, ${ }^{174}$

K. Lamichhane, ${ }^{174}$ S. W. Lee, ${ }^{174}$ T. Libeiro, ${ }^{174}$ T. Peltola, ${ }^{174}$ S. Undleeb, ${ }^{174}$ I. Volobouev, ${ }^{174}$ Z. Wang, ${ }^{174}$ S. Greene, ${ }^{175}$ A. Gurrola,${ }^{175}$ R. Janjam, ${ }^{175}$ W. Johns,${ }^{175}$ C. Maguire, ${ }^{175}$ A. Melo, ${ }^{175}$ H. Ni ${ }^{175}$ P. Sheldon,${ }^{175}$ S. Tuo, ${ }^{175}$ J. Velkovska, ${ }^{175}$ Q. Xu, ${ }^{175}$ M. W. Arenton, ${ }^{176}$ P. Barria, ${ }^{176}$ B. Cox, ${ }^{176}$ R. Hirosky, ${ }^{176}$ A. Ledovskoy, ${ }^{176} \mathrm{H}$. Li, ${ }^{176}$ C. Neu, ${ }^{176}$ T. Sinthuprasith, ${ }^{176}$ X. Sun, ${ }^{176}$ Y. Wang, ${ }^{176}$ E. Wolfe, ${ }^{176}$ F. Xia, ${ }^{176}$ C. Clarke, ${ }^{177}$ R. Harr, ${ }^{177}$ P. E. Karchin, ${ }^{177}$ J. Sturdy, ${ }^{177}$ S. Zaleski, ${ }^{177}$ J. Buchanan, ${ }^{178}$ C. Caillol, ${ }^{178}$ S. Dasu, ${ }^{178}$ L. Dodd, ${ }^{178}$ S. Duric,${ }^{178}$ B. Gomber, ${ }^{178}$ M. Grothe,${ }^{178}$ M. Herndon, ${ }^{178}$ A. Hervé, ${ }^{178}$ U. Hussain, ${ }^{178}$ P. Klabbers, ${ }^{178}$ A. Lanaro, ${ }^{178}$ A. Levine, ${ }^{178}$ K. Long, ${ }^{178}$ R. Loveless,${ }^{178}$ G. A. Pierro, ${ }^{178}$ G. Polese, ${ }^{178}$ T. Ruggles, ${ }^{178}$ A. Savin, ${ }^{178}$ N. Smith,${ }^{178}$ W. H. Smith, ${ }^{178}$ D. Taylor, ${ }^{178}$ and N. Woods ${ }^{178}$

(CMS Collaboration)

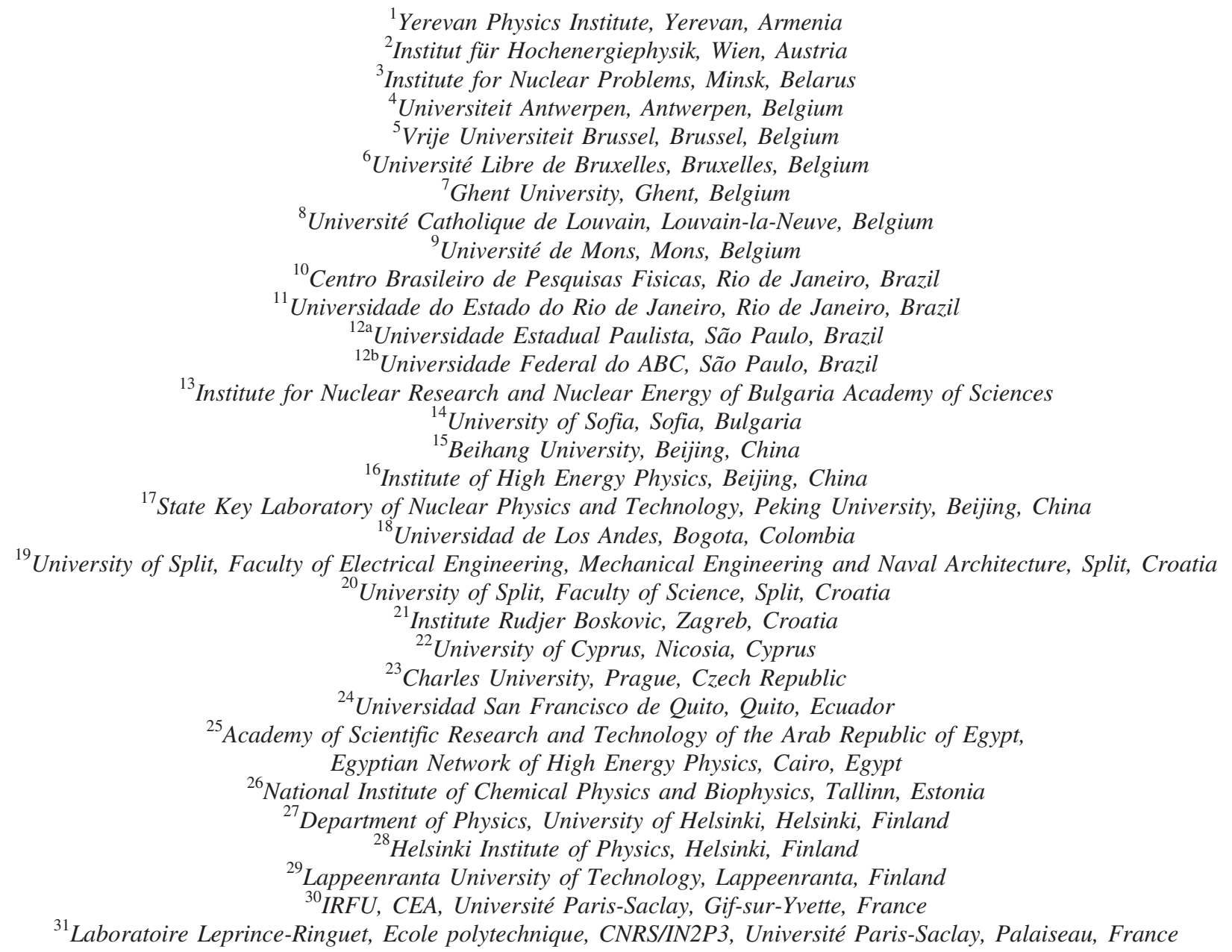


${ }^{32}$ Université de Strasbourg, CNRS, IPHC UMR 7178, F-67000 Strasbourg, France

${ }^{33}$ Centre de Calcul de l'Institut National de Physique Nucleaire et de Physique des Particules, CNRS/IN2P3, Villeurbanne, France

${ }^{34}$ Université de Lyon, Université Claude Bernard Lyon 1, CNRS-IN2P3, Institut de Physique Nucléaire de Lyon, Villeurbanne, France

${ }^{35}$ Georgian Technical University, Tbilisi, Georgia

${ }^{36}$ Tbilisi State University, Tbilisi, Georgia

${ }^{37}$ RWTH Aachen University, I. Physikalisches Institut, Aachen, Germany

${ }^{38}$ RWTH Aachen University, III. Physikalisches Institut A, Aachen, Germany

${ }^{39}$ RWTH Aachen University, III. Physikalisches Institut B, Aachen, Germany

${ }^{40}$ Deutsches Elektronen-Synchrotron, Hamburg, Germany

${ }^{41}$ University of Hamburg, Hamburg, Germany

${ }^{42}$ Institut für Experimentelle Kernphysik, Karlsruhe, Germany

${ }^{43}$ Institute of Nuclear and Particle Physics (INPP), NCSR Demokritos, Aghia Paraskevi, Greece

${ }^{44}$ National and Kapodistrian University of Athens, Athens, Greece

${ }^{45}$ University of Ioánnina, Ioánnina, Greece

${ }^{46}$ MTA-ELTE Lendület CMS Particle and Nuclear Physics Group, Eötvös Loránd University, Budapest, Hungary

${ }^{47}$ Wigner Research Centre for Physics, Budapest, Hungary

${ }^{48}$ Institute of Nuclear Research ATOMKI, Debrecen, Hungary

${ }^{49}$ Institute of Physics, University of Debrecen, Debrecen, Hungary

${ }^{50}$ Indian Institute of Science (IISc), Bangalore, India

${ }^{51}$ National Institute of Science Education and Research, Bhubaneswar, India

${ }^{52}$ Panjab University, Chandigarh, India

${ }^{53}$ University of Delhi, Delhi, India

${ }^{54}$ Saha Institute of Nuclear Physics, HBNI, Kolkata,India

${ }^{55}$ Indian Institute of Technology Madras, Madras, India

${ }^{56}$ Bhabha Atomic Research Centre, Mumbai, India

${ }^{57}$ Tata Institute of Fundamental Research-A, Mumbai, India

${ }^{58}$ Tata Institute of Fundamental Research-B, Mumbai, India

${ }^{59}$ Indian Institute of Science Education and Research (IISER), Pune, India

${ }^{60}$ Institute for Research in Fundamental Sciences (IPM), Tehran, Iran

${ }^{61}$ University College Dublin, Dublin, Ireland

${ }^{62 \mathrm{a}}$ INFN Sezione di Bari, Bari, Italy

${ }^{62 \mathrm{~b}}$ Università di Bari, Bari, Italy

${ }^{62 \mathrm{c}}$ Politecnico di Bari, Bari, Italy

${ }^{63 \mathrm{a}}$ INFN Sezione di Bologna, Bologna, Italy

${ }^{63 \mathrm{~b}}$ Università di Bologna, Bologna, Italy

${ }^{64 a}$ INFN Sezione di Catania, Catania, Italy

${ }^{64 \mathrm{~b}}$ Università di Catania, Catania, Italy

${ }^{65 a}$ INFN Sezione di Firenze, Firenze, Italy

${ }^{65 \mathrm{~b}}$ Università di Firenze, Firenze, Italy

${ }^{66}$ INFN Laboratori Nazionali di Frascati, Frascati, Italy

${ }^{67 a}$ INFN Sezione di Genova, Genova, Italy

${ }^{67 \mathrm{~b}}$ Università di Genova, Genova, Italy

${ }^{68 \mathrm{a}}$ INFN Sezione di Milano-Bicocca

${ }^{68 \mathrm{~b}}$ Università di Milano-Bicocca

${ }^{69 a}$ INFN Sezione di Napoli, Napoli, Italy

${ }^{69 \mathrm{~b}}$ Università di Napoli 'Federico II', Napoli, Italy

${ }^{69 \mathrm{c}}$ Università della Basilicata, Roma, Italy

${ }^{69 \mathrm{~d}}$ Università G. Marconi, Roma, Italy

${ }^{70 a}$ INFN Sezione di Padova, Padova, Italy

${ }^{70 \mathrm{~b}}$ Università di Padova, Padova, Italy

${ }^{70 \mathrm{c}}$ Università di Trento, Trento, Italy

${ }^{71 a}$ INFN Sezione di Pavia, Pavia, Italy

${ }^{71 b}$ Università di Pavia, Pavia, Italy

${ }^{72 a}$ INFN Sezione di Perugia, Perugia, Italy

${ }^{72 \mathrm{~b}}$ Università di Perugia, Perugia, Italy

${ }^{73 a}$ INFN Sezione di Pisa, Pisa, Italy

${ }^{73 b}$ Università di Pisa, Pisa, Italy

${ }^{73 \mathrm{c}}$ Scuola Normale Superiore di Pisa, Pisa, Italy

${ }^{74 a}$ INFN Sezione di Roma

${ }^{74 \mathrm{~b}}$ Sapienza Università di Roma 
${ }^{75 a}$ INFN Sezione di Torino, Torino, Italy

${ }^{75 b}$ Università di Torino, Torino, Italy

${ }^{75 \mathrm{c}}$ Università del Piemonte Orientale, Novara, Italy

${ }^{76 a}$ INFN Sezione di Trieste, Trieste, Italy

${ }^{76 \mathrm{~b}}$ Università di Trieste, Trieste, Italy

${ }^{77}$ Kyungpook National University, Daegu, Korea

${ }^{78}$ Chonbuk National University, Jeonju, Korea

${ }^{79}$ Chonnam National University, Institute for Universe and Elementary Particles, Kwangju, Korea

${ }^{80}$ Hanyang University, Seoul, Korea

${ }^{81}$ Korea University, Seoul, Korea

${ }^{82}$ Seoul National University, Seoul, Korea

${ }^{83}$ University of Seoul, Seoul, Korea

${ }^{84}$ Sungkyunkwan University, Suwon, Korea

${ }^{85}$ Vilnius University, Vilnius, Lithuania

${ }^{86}$ National Centre for Particle Physics, Universiti Malaya, Kuala Lumpur, Malaysia

${ }^{87}$ Centro de Investigacion y de Estudios Avanzados del IPN, Mexico City, Mexico

${ }^{88}$ Universidad Iberoamericana, Mexico City, Mexico

${ }^{89}$ Benemerita Universidad Autonoma de Puebla, Puebla, Mexico

${ }^{90}$ Universidad Autónoma de San Luis Potosí, San Luis Potosí, Mexico

${ }^{91}$ University of Auckland, Auckland, New Zealand

${ }^{92}$ University of Canterbury, Christchurch, New Zealand

${ }^{93}$ National Centre for Physics, Quaid-I-Azam University, Islamabad, Pakistan

${ }^{94}$ National Centre for Nuclear Research, Swierk, Poland

${ }^{95}$ Institute of Experimental Physics, Faculty of Physics, University of Warsaw, Warsaw, Poland

${ }^{96}$ Laboratório de Instrumentação e Física Experimental de Partículas, Lisboa, Portugal

${ }^{97}$ Joint Institute for Nuclear Research, Dubna, Russia

${ }^{98}$ Petersburg Nuclear Physics Institute, Gatchina (St. Petersburg), Russia

${ }^{99}$ Institute for Nuclear Research, Moscow, Russia

${ }^{100}$ Institute for Theoretical and Experimental Physics, Moscow, Russia

${ }^{101}$ Moscow Institute of Physics and Technology, Moscow, Russia

${ }^{102}$ National Research Nuclear University 'Moscow Engineering Physics Institute' (MEPhI), Moscow, Russia

${ }^{103}$ P.N. Lebedev Physical Institute, Moscow, Russia

${ }^{104}$ Skobeltsyn Institute of Nuclear Physics, Lomonosov Moscow State University, Moscow, Russia

${ }^{105}$ Novosibirsk State University (NSU), Novosibirsk, Russia

${ }^{106}$ State Research Center of Russian Federation, Institute for High Energy Physics, Protvino, Russia

${ }^{107}$ University of Belgrade, Faculty of Physics and Vinca Institute of Nuclear Sciences, Belgrade, Serbia

${ }^{108}$ Centro de Investigaciones Energéticas Medioambientales y Tecnológicas (CIEMAT), Madrid, Spain

${ }^{109}$ Universidad Autónoma de Madrid, Madrid, Spain

${ }^{110}$ Universidad de Oviedo, Oviedo, Spain

${ }^{111}$ Instituto de Física de Cantabria (IFCA), CSIC-Universidad de Cantabria, Santander, Spain

${ }^{112}$ CERN, European Organization for Nuclear Research, Geneva, Switzerland

${ }^{113}$ Paul Scherrer Institut, Villigen, Switzerland

${ }^{114}$ Institute for Particle Physics, ETH Zurich, Zurich, Switzerland

${ }^{115}$ Universität Zürich, Zurich, Switzerland

${ }^{116}$ National Central University, Chung-Li, Taiwan

${ }^{117}$ National Taiwan University (NTU), Taipei, Taiwan

${ }^{118}$ Chulalongkorn University, Faculty of Science, Department of Physics, Bangkok, Thailand

${ }^{119}$ Cukurova University, Physics Department, Science and Art Faculty, Adana, Turkey

${ }^{120}$ Middle East Technical University, Physics Department, Ankara, Turkey

${ }^{121}$ Bogazici University, Istanbul, Turkey

${ }^{122}$ Istanbul Technical University, Istanbul, Turkey

${ }^{123}$ Institute for Scintillation Materials of National Academy of Science of Ukraine, Kharkov, Ukraine

${ }^{124}$ National Scientific Center, Kharkov Institute of Physics and Technology, Kharkov, Ukraine

${ }^{125}$ University of Bristol, Bristol, United Kingdom

${ }^{126}$ Rutherford Appleton Laboratory, Didcot, United Kingdom

${ }^{127}$ Imperial College, London, United Kingdom

${ }^{128}$ Brunel University, Uxbridge, United Kingdom

${ }^{129}$ Baylor University, Waco, Texas, USA

${ }^{130}$ Catholic University of America, Washington DC, USA 
${ }^{131}$ The University of Alabama, Tuscaloosa, Alabama, USA

${ }^{132}$ Boston University, Boston, Massachusetts, USA

${ }^{133}$ Brown University, Providence, Rhode Island, USA

${ }^{134}$ University of California, Davis, Davis, California, USA

${ }^{135}$ University of California, Los Angeles, California, USA

${ }^{136}$ University of California, Riverside, Riverside, USA

${ }^{137}$ University of California, San Diego, La Jolla, USA

${ }^{138}$ University of California, Santa Barbara - Department of Physics, Santa Barbara, California, USA

${ }^{139}$ California Institute of Technology, Pasadena, California, USA

${ }^{140}$ Carnegie Mellon University, Pittsburgh, Pennsylvania, USA

${ }^{141}$ University of Colorado Boulder, Boulder, Colorado, USA

${ }^{142}$ Cornell University, Ithaca, New York, USA

${ }^{143}$ Fermi National Accelerator Laboratory, Batavia, Illinois, USA

${ }^{144}$ University of Florida, Gainesville, Florida, USA

${ }^{145}$ Florida International University, Miami, Florida, USA

${ }^{146}$ Florida State University, Tallahassee, Florida, USA

${ }^{147}$ Florida Institute of Technology, Melbourne, Florida, USA

${ }^{148}$ University of Illinois at Chicago (UIC), Chicago, Illinois, USA

${ }^{149}$ The University of Iowa, Iowa City, Iowa, USA

${ }^{150}$ Johns Hopkins University, Baltimore, Maryland, USA

${ }^{151}$ The University of Kansas, Lawrence, Kansas, USA

${ }^{152}$ Kansas State University, Manhattan, Kansas, USA

${ }^{153}$ Lawrence Livermore National Laboratory, Livermore, California, USA

${ }^{154}$ University of Maryland, College Park, Maryland, USA

${ }^{155}$ Massachusetts Institute of Technology, Cambridge, Massachusetts, USA

${ }^{156}$ University of Minnesota, Minneapolis, Minnesota, USA

${ }^{157}$ University of Mississippi, Oxford, Mississippi, USA

${ }^{158}$ University of Nebraska-Lincoln, Lincoln, Nebraska, USA

${ }^{159}$ State University of New York at Buffalo, Buffalo, New York, USA

${ }^{160}$ Northeastern University, Boston, Massachusetts, USA

${ }^{161}$ Northwestern University, Evanston, Illinois, USA

${ }^{162}$ University of Notre Dame, Notre Dame, Indiana, USA

${ }^{163}$ The Ohio State University, Columbus, Ohio, USA

${ }^{164}$ Princeton University, Princeton, New Jersey, USA

${ }^{165}$ University of Puerto Rico, Mayaguez, Puerto Rico, USA

${ }^{166}$ Purdue University, West Lafayette, Indiana, USA

${ }^{167}$ Purdue University Northwest, Hammond, Indiana, USA

${ }^{168}$ Rice University, Houston, Texas, USA

${ }^{169}$ University of Rochester, Rochester, New York, USA

${ }^{170}$ The Rockefeller University, New York, New York, USA

${ }^{171}$ Rutgers, The State University of New Jersey, Piscataway, New Jersey, USA

${ }^{172}$ University of Tennessee, Knoxville, Tennessee, USA

${ }^{173}$ Texas A\&M University, College Station, Texas, USA

${ }^{174}$ Texas Tech University, Lubbock, Texas, USA

${ }^{175}$ Vanderbilt University, Nashville, Tennessee, USA

${ }^{176}$ University of Virginia, Charlottesville, Virginia, USA

${ }^{177}$ Wayne State University, Detroit, Michigan, USA

${ }^{178}$ University of Wisconsin - Madison, Madison, Wisconsin, USA

${ }^{\mathrm{a}}$ Deceased.

${ }^{\mathrm{b}}$ Also at Vienna University of Technology, Vienna, Austria.

${ }^{c}$ Also at State Key Laboratory of Nuclear Physics and Technology, Peking University, Beijing, China.

${ }^{\mathrm{d}}$ Also at Universidade Estadual de Campinas, Campinas, Brazil.

${ }^{\mathrm{e}}$ Also at Universidade Federal de Pelotas, Pelotas, Brazil.

${ }^{\mathrm{f}}$ Also at Université Libre de Bruxelles, Bruxelles, Belgium.

${ }^{g}$ Also at Joint Institute for Nuclear Research, Dubna, Russia.

${ }^{\mathrm{h}}$ Also at Helwan University, Cairo, Egypt.

${ }^{\mathrm{i}}$ Also at Zewail City of Science and Technology, Zewail, Egypt.

${ }^{\mathrm{j}}$ Also at Fayoum University, El-Fayoum, Egypt. 
${ }^{\mathrm{k}}$ Also at British University in Egypt, Cairo, Egypt.

${ }^{1}$ Also at Ain Shams University, Cairo, Egypt.

${ }^{\mathrm{m}}$ Also at Université de Haute Alsace, Mulhouse, France.

${ }^{\mathrm{n}}$ Also at Skobeltsyn Institute of Nuclear Physics, Lomonosov Moscow State University, Moscow, Russia.

${ }^{\circ}$ Also at Tbilisi State University, Tbilisi, Georgia.

${ }^{\mathrm{p}}$ Also at CERN, European Organization for Nuclear Research, Geneva, Switzerland.

${ }^{\mathrm{q}}$ Also at RWTH Aachen University, III. Physikalisches Institut A, Aachen, Germany.

${ }^{\mathrm{r}}$ Also at University of Hamburg, Hamburg, Germany.

${ }^{\mathrm{s}}$ Also at Brandenburg University of Technology, Cottbus, Germany.

${ }^{t}$ Also at Institute of Nuclear Research ATOMKI, Debrecen, Hungary.

"Also at MTA-ELTE Lendület CMS Particle and Nuclear Physics Group, Eötvös Loránd University, Budapest, Hungary.

${ }^{v}$ Also at Institute of Physics, University of Debrecen, Debrecen, Hungary.

${ }^{\mathrm{w}}$ Also at IIT Bhubaneswar, Bhubaneswar, India.

${ }^{\mathrm{x}}$ Also at Institute of Physics, Bhubaneswar, India.

${ }^{y}$ Also at University of Visva-Bharati, Santiniketan, India.

${ }^{\mathrm{z}}$ Also at University of Ruhuna, Matara, Sri Lanka.

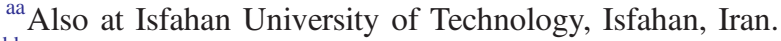

${ }^{\text {bb }}$ Also at Yazd University, Yazd, Iran.

${ }^{c c}$ Also at Plasma Physics Research Center, Science and Research Branch, Islamic Azad University, Tehran, Iran.

${ }^{\mathrm{dd}}$ Also at Università degli Studi di Siena, Siena, Italy.

${ }^{e e}$ Also at INFN Sezione di Milano-Bicocca, Università di Milano-Bicocca, Milano, Italy.

${ }^{\mathrm{ff}}$ Also at Purdue University, West Lafayette, USA.

${ }^{\mathrm{gg}}$ Also at International Islamic University of Malaysia, Kuala Lumpur, Malaysia.

${ }^{\text {hh }}$ Also at Malaysian Nuclear Agency, MOSTI, Kajang, Malaysia.

ii Also at Consejo Nacional de Ciencia y Tecnología, Mexico city, Mexico.

${ }^{\mathrm{jj}}$ Also at Warsaw University of Technology, Institute of Electronic Systems, Warsaw, Poland.

${ }^{\mathrm{kk}}$ Also at Institute for Nuclear Research, Moscow, Russia.

"Also at National Research Nuclear University 'Moscow Engineering Physics Institute' (MEPhI), Moscow, Russia.

${ }^{\mathrm{mm}}$ Also at St. Petersburg State Polytechnical University, St. Petersburg, Russia.

${ }^{\mathrm{nn}}$ Also at University of Florida, Gainesville, USA.

${ }^{00}$ Also at P.N. Lebedev Physical Institute, Moscow, Russia.

${ }^{\mathrm{pp}}$ Also at California Institute of Technology, Pasadena, USA.

${ }^{\mathrm{qq}}$ Also at Budker Institute of Nuclear Physics, Novosibirsk, Russia.

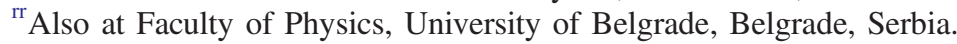

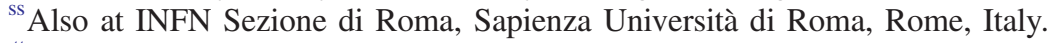

"Also at University of Belgrade, Faculty of Physics and Vinca Institute of Nuclear Sciences, Belgrade, Serbia.

${ }^{\text {uu }}$ Also at Scuola Normale e Sezione dell'INFN, Pisa, Italy.

${ }^{\mathrm{vv}}$ Also at National and Kapodistrian University of Athens, Athens, Greece.

${ }^{w w}$ Also at Riga Technical University, Riga, Latvia.

${ }^{\mathrm{xx}}$ Also at Institute for Theoretical and Experimental Physics, Moscow, Russia.

${ }^{y y}$ Also at Stefan Meyer Institute for Subatomic Physics.

${ }^{\mathrm{zz}}$ Also at Istanbul University, Faculty of Science, Istanbul, Turkey.

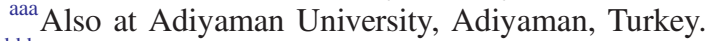

${ }^{b b b}$ Also at Istanbul Aydin University, Istanbul, Turkey.

${ }^{c c c}$ Also at Mersin University, Mersin, Turkey.

ddd Also at Cag University, Mersin, Turkey.

${ }^{e e e}$ Also at Piri Reis University, Istanbul, Turkey.

${ }^{\text {fff }}$ Also at Gaziosmanpasa University, Tokat, Turkey.

ggg Also at Izmir Institute of Technology, Izmir, Turkey.

${ }^{\text {hhh }}$ Also at Necmettin Erbakan University, Konya, Turkey.

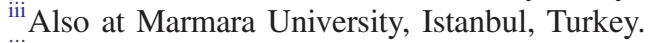

${ }^{i j j}$ Also at Kafkas University, Kars, Turkey.

${ }^{k k k}$ Also at Istanbul Bilgi University, Istanbul, Turkey.

${ }^{111}$ Also at Rutherford Appleton Laboratory, Didcot, United Kingdom.

${ }^{\mathrm{mmm}}$ Also at School of Physics and Astronomy, University of Southampton, Southampton, United Kingdom.

${ }^{n n n}$ Also at Instituto de Astrofísica de Canarias, La Laguna, Spain.

${ }^{\circ o o}$ Also at Utah Valley University, Orem, USA.

${ }^{\mathrm{ppp}}$ Also at Beykent University.

${ }^{\mathrm{qqq}}$ Also at Bingol University, Bingol, Turkey.

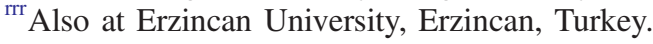


${ }^{\text {sss }}$ Also at Sinop University, Sinop, Turkey.

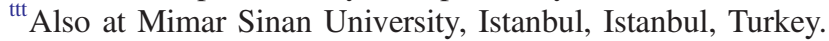

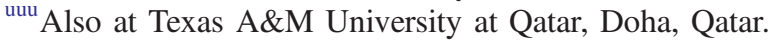

${ }^{v v v}$ Also at Kyungpook National University, Daegu, Korea. 